\title{
Construction and verification of the transcriptional regulatory response network of Streptococcus mutans upon treatment with the biofilm inhibitor carolacton
}

Padhmanand Sudhakar ${ }^{1 \dagger}$, Michael Reck ${ }^{2 \dagger}$, Wei Wang ${ }^{1}$, Feng Q He ${ }^{3}$, Irene W Dobler ${ }^{2^{*}}$ and An-Ping Zeng ${ }^{1 *}$

\begin{abstract}
Background: Carolacton is a newly identified secondary metabolite causing altered cell morphology and death of Streptococcus mutans biofilm cells. To unravel key regulators mediating these effects, the transcriptional regulatory response network of $S$. mutans biofilms upon carolacton treatment was constructed and analyzed. A systems biological approach integrating time-resolved transcriptomic data, reverse engineering, transcription factor binding sites, and experimental validation was carried out.

Results: The co-expression response network constructed from transcriptomic data using the reverse engineering algorithm called the Trend Correlation method consisted of 8284 gene pairs. The regulatory response network inferred by superimposing transcription factor binding site information into the co-expression network comprised 329 putative transcriptional regulatory interactions and could be classified into 27 sub-networks each co-regulated by a transcription factor. These sub-networks were significantly enriched with genes sharing common functions. The regulatory response network displayed global hierarchy and network motifs as observed in model organisms. The sub-networks modulated by the pyrimidine biosynthesis regulator PyrR, the glutamine synthetase repressor $\mathrm{GlnR}$, the cysteine metabolism regulator CysR, global regulators CcpA and CodY and the two component system response regulators VicR and MbrC among others could putatively be related to the physiological effect of carolacton. The predicted interactions from the regulatory network between MbrC, known to be involved in cell envelope stress response, and the murMN-SMU_718c genes encoding peptidoglycan biosynthetic enzymes were experimentally confirmed using Electro Mobility Shift Assays. Furthermore, gene deletion mutants of five predicted key regulators from the response networks were constructed and their sensitivities towards carolacton were investigated. Deletion of cysR, the node having the highest connectivity among the regulators chosen from the regulatory network, resulted in a mutant which was insensitive to carolacton thus demonstrating not only the essentiality of cysR for the response of $S$. mutans biofilms to carolacton but also the relevance of the predicted network.
\end{abstract}

Conclusion: The network approach used in this study revealed important regulators and interactions as part of the response mechanisms of $S$. mutans biofilm cells to carolacton. It also opens a door for further studies into novel drug targets against streptococci.

Keywords: Streptococcus mutans, Caries, Biofilm inhibitor, Carolacton, Transcriptome, Reverse engineering, Transcriptional regulatory network, Key regulators, Network verification

\footnotetext{
* Correspondence: iwd@helmholtz-hzi.de; aze@tuhh.de

${ }^{\dagger}$ Equal contributors

${ }^{2}$ Research Group Microbial Communication, Helmholtz Center for Infection

Research, Inhoffenstrasse 7, 38124 Braunschweig, Germany

'Institute of Bioprocess and Biosystems Engineering, Hamburg University of

Technology, 21073 Hamburg, Germany

Full list of author information is available at the end of the article
} 


\section{Background}

Streptococcus mutans is an oral pathogen, which along with other closely related streptococci called the mutans streptococci, plays an important role in the formation of caries and tooth decay in humans. This is attributed to its ability to form biofilms which are generally difficult or impossible to eradicate by antibiotic therapy because biofilm cells are resistant to antibiotics [1,2].

Recently, it was shown that carolacton, a secondary metabolite from the myxobacterial species Sorangium cellulosum has a high inhibitory activity against actively growing $S$. mutans biofilm cells resulting in changes in cell morphology, elongation of cell chains, membrane damage and death of a part of the population. Carolacton was also found to induce a dose-dependent damage of $S$. mutans biofilms over a wide concentration range resembling a sigmoid dose-response curve [3]. Carolacton inhibits $S$. mutans biofilms even at nanomolecular concentrations [3] implying that it primarily targets molecular entities which are present only as a few copies per cell. In this regard, carolacton is very similar to compounds which target cellular signaling networks [4] rather than directly targeting particular enzymes in pathways associated with vital processes such as protein, DNA/RNA synthesis, cell division etc.

To decipher the genes whose expression is affected by carolacton, a time resolved transcriptome analysis of S. mutans biofilms after carolacton treatment was carried out by Reck et al. [5]. Results from the study indicate that carolacton affects changes in the expression of genes related to biofilm formation, autolysis, pyrimidine and histidine metabolism, cell shape and cell division in addition to two component systems (TCSs). Among the TCSs, the vicRK system shows an immediate strong downregulation, while the comDE system controlling competence development through quorum sensing [6,7] is upregulated. A deletion mutant for the histidine kinase encoding gene vicK which responds instantaneously to carolacton treatment together with all the vicR-coexpressed genes, was similarly tested for carolacton-sensitivity, but since the vicK mutant showed very poor biofilm growth, further inferences could not be made. VicR is an essential gene and cannot be deleted in S. mutans [8]. Meanwhile, the $S$. mutans eukaryotic-like serine-threonine protein kinase $p k n B$ whose ortholog has been shown to be a master regulator of virulence in S. pneumoniae [9] displayed no observable changes in transcription [5]. However, when $p k n B$ is knocked out, it results in a carolacton-insensitive mutant [5]. These data show that even though the physiological and genetic responses of carolacton-treated $S$. mutans biofilm cells are known, the underlying network which orchestrates the expression of affected genes in response to carolacton still remains a mystery. This calls for an effort to uncover the effect of carolacton at the network level. Although Reck et al. [5] have already measured the temporal progression of the $S$. mutans transcriptome in response to carolacton, their dataset is characterized by a small number of sampling points (five) and large time intervals, which does not allow for a reliable network inference. Hence, an extended time-series transcriptome is required encompassing a higher number of sampling points with relatively short intervals and was carried out in the current study.

Reverse engineering based network reconstruction methods have widely been used to infer genetic networks from gene expression data measured most commonly using cDNA microarrays. Excellent reviews about genetic network reconstruction from expression data have been published [10]. In order to capture the network level events of biological systems upon exposure to various stimuli, reverse engineering methods have been developed to infer underlying networks from time series expression data in addition to static data [11]. For instance, methods such as Time Series Network Identification (TSNI) were used for inferring co-expression networks and compound mode of action from time-series gene expression data following interventions [12]. Although the inferred coexpression network using TSNI involved only nine genes of the E. coli DNA-damage response pathway, it is seen as one of the first attempts in network reconstruction for determining the compound mode of action from a time series transcriptome. Further tools and algorithms were also developed to deal with whole genome network inference $[13,14]$, but the gene-gene interactions in such inferred networks were either undirected (no assigned causality) or carry only a statistical probability of causality. Despite lacking directionality, undirected co-expression networks have nevertheless been used to infer critical genes and networks involved in T cell functions [15].

Meanwhile, whole genome directed networks were inferred by using combinatorial methodologies [16] incorporating gene-gene interactions from reverse engineering algorithms with biological information such as data on binding motifs and promoter elements [17], functional genomics [18], genome annotation [19] and transcription factor activities [20]. Although non-exhaustive due to the lack of sufficient biological information, genome wide transcriptional regulatory networks have nevertheless been constructed from heterogeneous data for a few organisms such as Escherichia coli [20], Saccharomyces cerevisiae [21], Bacillus subtilis [17], Candida albicans [22], Streptomyces coelicolor [18], Halobacterium NRC-1 [19], Pseudomonas aeruginosa [23] and others. Despite the advances made in the field of genome-wide regulatory network reconstruction, most of these attempts have been carried out for model or standard organisms (for which ample biological information exists) such as E. coli, S. cerevisiae, B. subtilis, $P$. aeruginosa $[17,20,23,24]$. Some progress has 
also been made in the case of human cells as shown by Basso et al. where human B cell gene regulatory interactions were inferred [25]. Hence, one of the major current challenges in the field is the network inference for less-studied organisms which are either pathogens or are industrially important.

Even though genetic networks have been inferred under drug treatment conditions for some organisms, most of them have either been limited to compounds and molecules whose targets and mode of action were already known and/or limited to model organisms and certain human cell lines. To the best of our knowledge, reconstruction of a contextual genome-scale regulatory network for the human caries pathogen $S$. mutans has not yet been carried out although studies focusing on genome-wide transcriptome profiling have been reported [5,26-29]. A workflow based on a combinatorial strategy was executed in an attempt to infer a genome-wide network for $S$. mutans biofilms under conditions of treatment with the biofilm inhibitor carolacton. The Trend Correlation method which has been used to construct co-expression networks of human T cells [15] was used for inferring the S. mutans co-expression network under carolacton treatment conditions using microarray data generated in this study. This was followed by the construction of a transcriptional regulatory response network (TRRN) by incorporating binding site information. From the TRRN, we detected sub-networks whose activation or repression could be related to the biological effect of carolacton. A key regulator was identified based on its connectivity in the regulatory network and when knocked out, rendered S. mutans biofilms insensitive to carolacton. Furthermore, tested interactions from the inferred regulatory response network were confirmed using in vitro interaction detection assays.

\section{Results and discussion}

Dynamic genome-wide transcriptome profiling

A high resolution transcriptomic time-course experiment with $S$. mutans biofilms (with 11 samples taken from 5 to 300 min post carolacton treatment) was performed to capture the temporal response over an extended period of time. Our time-series data included results from a sample taken at 5 min post carolacton treatment and is expected to identify genes whose expression patterns were immediately affected by carolacton. A total of 772 GTAAs (genes corresponding to transcripts with altered abundances) or differentially expressed genes were identified at a log-fold change $>=0.8$.

Enrichment analysis with respect to various categories such as biological functional classes and KEGG metabolic pathways was performed for the up- and downregulated GTAAs at every time point in order to gain an overview of the various categories of genes affected by carolacton (Additional file 1). The results from the enrichment analysis of upregulated GTAAs indicated that transcripts from the pyrimidine ribonucleotide biosynthesis ( $\mathrm{P}$-value $<=4.09 \mathrm{E}-18$ ), nucleosides, purines and pyrimidines' transport (P-value $<=2.45 \mathrm{E}-2)$, and metabolism of alanine, aspartate and glutamate (P-value $<=7.2 \mathrm{E}-4)$ were most significantly over-represented at both $5 \mathrm{~min}$ and 20 min but not at $40 \mathrm{~min}$. These observations suggest a possible specific role of pyrimidine biosynthesis and metabolism in the initial response of $S$. mutans biofilms to carolacton. At later time points however, many more categories were found to be over-represented among the set of upregulated GTAAs due to probable cascade effects.

Enrichment analysis of the downregulated GTAAs at 20 min revealed categories such as glutamate biosynthesis, TCA cycle, anaerobic energy metabolism, glyoxylate and dicarboxylate metabolism, nitrogen metabolism as well the signal transduction related two component systems (TCSs). TCSs impart various virulence characteristics to $S$. mutans by sensing and responding to various signals related to stress, thus enabling it to survive and tolerate unfavourable conditions [30,31]. The transcriptional downregulation of TCS genes could indicate a possible effect of carolacton having a repressive effect on the signaling mechanisms of S. mutans. In general, downregulated GTAAs in the early part of the response were enriched with categories corresponding to the processes of signaling, energy and amino acid metabolism. The set of downregulated GTAAs in the later phases after 20 min was also found to be enriched with many categories over the course of the experiment. Interestingly, the alanine, aspartate and glutamate metabolic pathways were found to be over-represented in both the up- and downregulated GTAA sets at the time point of 20 min after carolacton treatment suggesting a dual regulation of such pathway enzymes in the initial response to carolacton. The information from our transcriptome data largely agrees with those from a previous study by Reck et al. [5] where only five sampling points with large intervals were used.

\section{Contextual co-expression network}

In order to identify co-expression relationships among genes immediately affected by carolacton, the co-expression network was confined to statistically significant optimal correlations which started either from 0,5 or $20 \mathrm{~min}$ after carolacton treatment (see Methods). The contextual co-expression network inferred according to the workflow shown in Figure 1 consisted of 8284 gene-gene co-expression relationships (see Additional file 2). 5430 (65.5\%) of the 8284 edges were characterized by time lagged coexpression relationships. 3959 (47.7\%) of the total number of co-expression relationships could be described as being inverted (opposite change trend in expression patterns) 


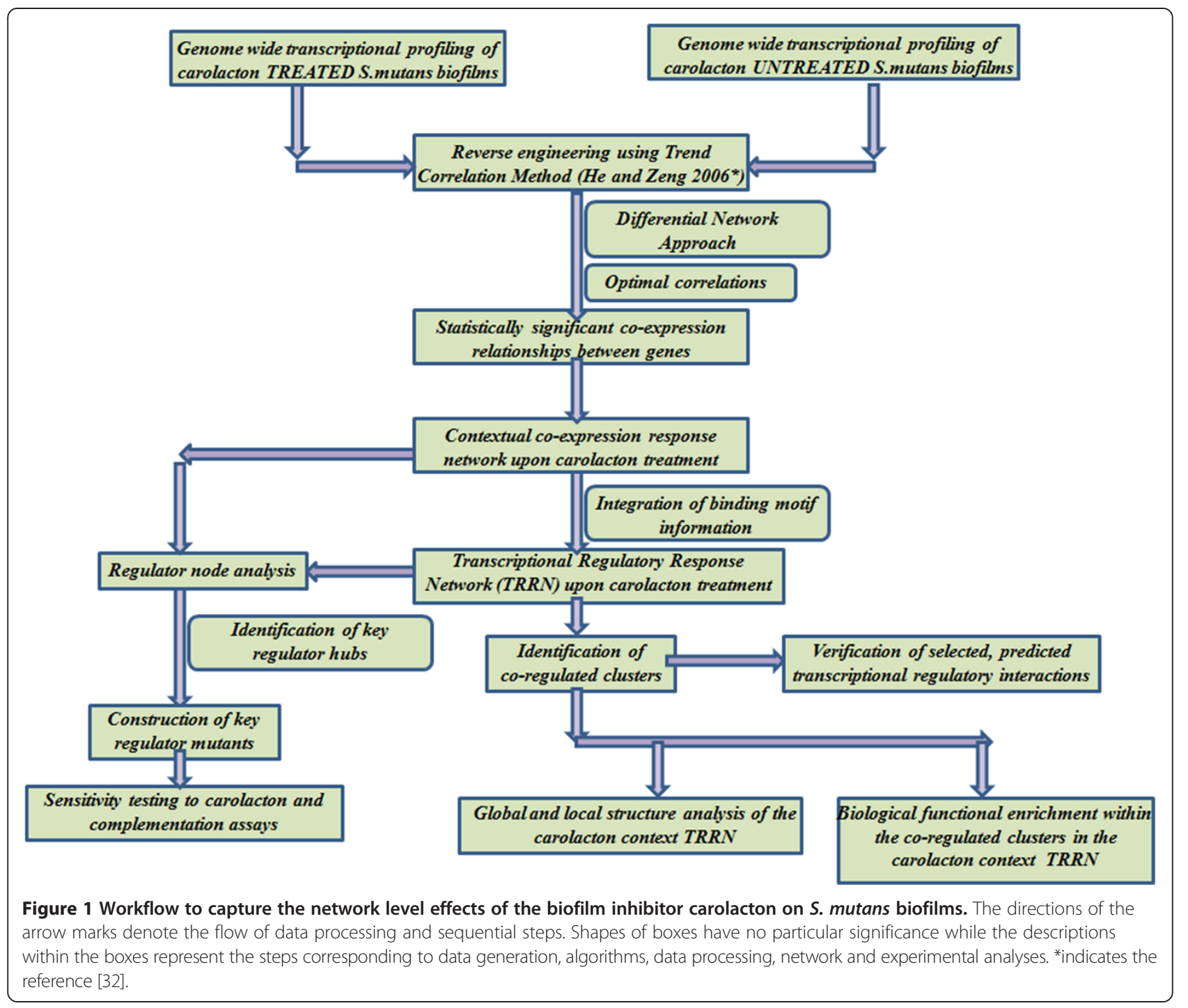

whereas the remaining were described as being positive (showing similar change trends).

\section{Regulator-target gene binding site map of S. mutans UA159}

The edges of the carolacton-context co-expression network derived from the dynamic transcriptome data are undirected. In other words, the mere presence of an edge between two genes does not necessarily represent a transcriptional regulatory relationship between them. Although time-lags between the co-expressed regulator and target gene(s) could possibly serve as a preliminary proof of direct/indirect transcriptional regulation [10], further evidence is required for at least assigning putative biological causality and for pursuing precise experimental validation of transcriptional regulatory relationships.

Among the more than 100 known or predicted transcriptional regulators in the $S$. mutans UA159 genome, only a few have been studied experimentally and characterized with respect to their DNA binding site(s). We compensated for the non-availability of experimentally verified transcriptional regulatory binding sites for the $S$. mutans transcriptional factors by applying comparative genomic approaches [33-35]. Binding site data for 44 transcriptional regulators were recovered from different sources such as experimental data from $S$. mutans itself or information from comparative genomic analysis. In cases where the collective number of binding sites inferred for a transcriptional regulator were fewer or equal to three, respective regulogic [33] sites from other species within the Streptococccus genus were retrieved. These were then used to construct PSSMs (Position Specific Scoring Matrices, see Methods) with improved predictive capacities. PSSMs were also constructed from regulogic sites available in the RegPrecise database [36] and from phylogenetic footprinting studies. An online 
Regulatory Sequence Analysis Tool (RSAT) termed matrix-quality [37] was used to objectively determine the optimal P-value for every PSSM (see Additional file 3). The PSSMs were then used to predict binding sites (at the corresponding optimal P-values) in the upstream regulatory regions of all the genes in the $S$. mutans UA159 genome. A total of 1397 unique regulator-target gene relationships (corresponding to 2056 predicted binding sites) based on binding site data alone corresponding to $44 \mathrm{~S}$. mutans UA159 transcriptional regulators were thus compiled (see Additional file 4). After integrating operon structure information, 2445 regulator-target gene relationships were found to comprise the final regulator-target gene binding site map. The global regulators CcpA (catabolite repressor protein) and CodY (global regulator of amino acid metabolism) together accounted for $\sim 29.22 \%$ of the 2445 regulator-target gene relationships based on binding sites.

\section{S. mutans Transcriptional Regulatory Response Network (TRRN) under carolacton treatment}

Combining inferred correlation relationships from the coexpression network and the regulator-binding site map resulted in 227 transcriptional regulatory relationships with predicted direct biological causality (see Additional file 5). After operon oriented adjustment (see Methods), the transcriptional response regulatory network (TRRN) (Figure 2) comprised 329 relationships among 307 genes, of which 37 were found to be transcription factors. Based on the predicted transcriptional regulatory connections, the TRRN genes could be organized into 27 co-regulated gene groups or sub-networks. A co-regulated gene group is defined as a set of genes which are putatively regulated by a common transcription factor. 10 of the 37 regulators were associated with predicted incoming connections only and were contained within the 27 sub-networks with different size distributions. The largest sub-network was that putatively modulated by CodY (which is a known global regulator [38]) and comprised 84 genes. The second largest sub-network consisted of 26 genes under the putative control of CysR (the cysteine metabolism regulatory protein encoded by SMU_852) followed by two sub-networks each containing 22 genes and predicted to be modulated by the essential TCS response regulator VicR and the global regulator CcpA, respectively.

The TRRN (Figure 2) represented a "waterfall-like" model with respect to its global topological structure and hierarchy $[39,40]$ with some regulators apart from modulating their own sub-networks also exerting indirect control over other sub-networks by putatively modulating the expression of the corresponding transcription factors. For example, it can be seen from the

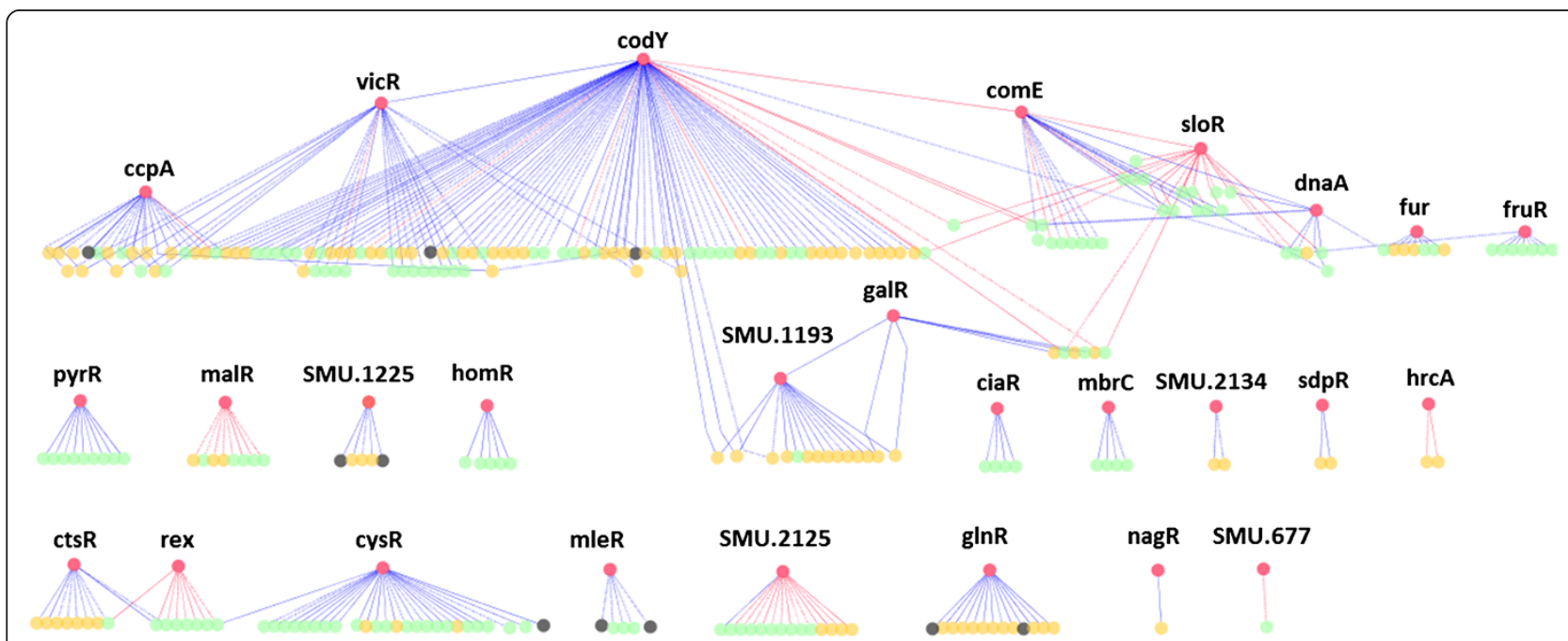

Figure 2 Topological view of the transcriptional regulatory response network (TRRN) of S. mutans biofilms upon carolacton treatment. The TRRN was inferred by overlaying the regulator-target gene binding site map onto the co-expression network. It consisted of 27 co-regulated gene groups or subnetworks each under the control of a transcription factor and comprised 329 regulatory interactions involving 307 genes. Some of the co-regulated gene groups overlap with each other as a result of genes modulated by more than one transcription factor. The 27 regulators with outgoing connections (marked with red circles) along with 10 other regulators only with incoming connections (marked with black circles) found to be among target genes within the sub-networks are indicated as well as non-regulator target genes (indicated by green circles). If the upstream regulatory regions of the target genes or their corresponding operons harbored multiple putative binding sites of its predicted regulator(s), then they are indicated by orange circles). In addition, the regulators with outgoing connections are also marked with their gene names above their respective nodes. Blue arrows indicate a positive (activation) relationship whereas red arrows stand for a negative (repression) effect. Connections always flow from top to bottom. Spatial positions of transcription factor nodes are manipulated so as to pictorially depict the possible hierarchies. The carolacton context TRRN shown herein is observed to be organized as a double layered hierarchy. The network was visualized using Cytoscape. 
inferred TRRN (Figure 2) that the global regulator CodY putatively regulated the expression of two distinct genes encoding the TCS response regulators VicR and ComE, thus potentially exerting an indirect influence on their sub-networks as well. Although global hierarchical organization was observed in the inferred TRRN, it was nevertheless confined only to a double-layered hierarchy compared to multi-layered hierarchies reported in the regulatory networks of well-studied organisms $[40,41]$. This could primarily be due to the lack of biological connectivity data, firstly as a result of the limited experimentally verified binding site information of $S$. mutans transcription factors. Secondly, the functional and/or evolutionary divergence of $S$. mutans transcription factors from their corresponding orthologs in standard organisms has a certain limiting influence on the comparative genomic based extrapolation of binding sites [33].

The overlap between co-regulated gene groups, which was previously observed in bacteria [42,43], is also a characteristic of the inferred TRRN. 13\% of the 307 TRRN genes were found to be under the putative control of more than one transcriptional regulator (implying an incoming degree $>1$ ). From a local network structure point of view, the nodes putatively controlled by just one regulator resemble single input motifs (SIM) whereas the nodes coregulated by several regulators form multiple input motifs (MIMs) [44]. Besides MIMs, overlap between co-regulated gene groups could be the manifestations of more complicated network motifs such as the feed forward loop (FFL) in which a regulator encoding gene is modulated by another regulator, both of which control the expression of a common gene [45]. If multiple genes are controlled by a single FFL, then it is termed as a multi-output FFL (MOFFL) [46]. Additional file 6 lists the various types of significant motifs identified (P-value $<=0.01$ after 10000 randomizations) in the carolacton context dependent TRRN among which were found FFLs including other motifs such as regulatory bi-fans [44] and their derivatives [47]. Local network topologies such as the motifs found here were consistent with previous observations in other organisms [44-46,48] and could be important for eliciting quick and flexible responses to stimuli [45]. Although the exact biological mechanisms which give rise to such motifs remain to be understood, these observations demonstrate not only the relevance of the constructed network but can also be further used for detailed dynamic quantitative modeling of smaller sub-systems.

\section{Biological context within co-regulated sub-networks in the TRRN}

The 27 co-regulated groups or sub-networks contained statistically significant over-represented (significance score $>=0$ ) functional categories such as biological functional classes, KEGG metabolic pathways and gene ontology terms (see Additional file 7). Figure 3 visualizes the TRRN enrichment data shown in Additional file 7. Put together, 22 enrichment events involving 6 KEGG metabolic pathways, 7 biological functional classes and 6 gene ontology terms were collectively identified within the 27 sub-networks. At least 7 cases were found in which the transcription factor or regulator belonged to the same functional category as that found to be over-represented in its target gene group, confirming that the constructed regulatory network is biologically meaningful. For instance, the sub-network co-regulated by the glutamine synthetase repressor GlnR known to control glutamine metabolism [49] in S. mutans was enriched with genes belonging to the functional categories "amino acid biosynthesis" and "nitrogen metabolism". On closer observation, most of the genes within the GlnR co-regulated subnetwork were related to glutamine metabolism. Genes belonging to the biological functional class of cell envelope metabolism were over-represented in the sub-network co-regulated by the TCS RR MbrC associated with cell envelope stress response [50]. Similarly, the genes putatively modulated by the pyrimidine regulatory protein PyrR contained an over-representation of pyrimidine metabolism genes. Several instances were also observed in which the functional category to which the regulator belonged to was different from that found to be over represented within the given sub-network (Figure 3, Additional file 7). Besides the above described intra- and inter categorical relationships between regulators and their sub-networks, the TRRN also contained regulatory relationships which were already verified by others (see Additional file 5).

\section{TRRN sub-networks co-regulated by global transcription factors}

The sub-networks of the global transcriptional regulators affecting central metabolism namely CodY and CcpA $[38,51]$ were among the top three sub-networks in terms of the number of genes. CcpA is reported to modulate sugar uptake and metabolism, carbon catabolite repression and expression of virulence related genes in S. mutans [51]. The treatment of $S$. mutans biofilms with carolacton caused an immediate downregulation of CcpA modulated genes (Additional file 8). The CcpA sub-network consisted of genes encoding components of two mannose specific phosphotransferase systems (ptnAC (manLM)-SMU_1879 and SMU_1960c-SMU_1961c) [52-54], as well as three ATP dependent transporter complexes (msmEFG, SMU_921SMU_922 and SMU_1315c-SMU_1317c) among others. While msmEFG is reportedly involved in the energy dependent transport of multiple sugars [55], the substrate specificities of the other two clusters are unknown. Subnetworks of other sugar-specific transcription factors such as the repressors GalR and FruR of galactose and fructose 

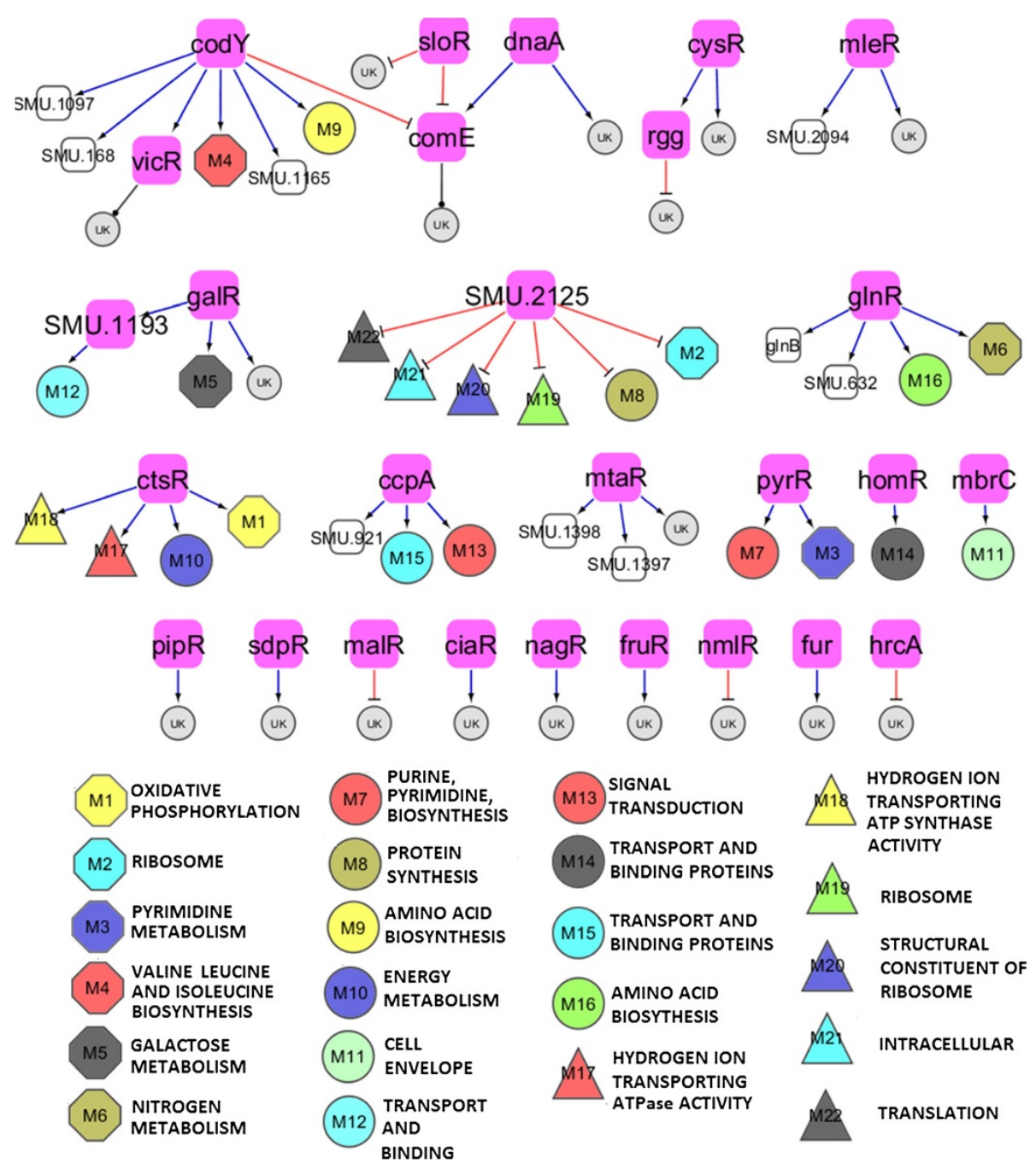

Figure 3 Categorical enrichment within the sub-networks comprising the $S$. mutans biofilms TRRN upon carolacton treatment. The co-regulated groups or sub-networks within the TRRN were found to be enriched with functional categories such as KEGG metabolic pathways, biological functional classes and gene ontology terms thus lending biological meaning to the inferred regulatory response network. Co-regulated groups denote sets of genes predicted to be commonly modulated by a transcription factor (indicated by filled pink rectangles). The regulatory response network also includes other transcriptional factors with only incoming connections (black rectangles in bold) and found to be among the co-regulated groups. Some of the co-regulated groups were found to be enriched with functional categories such as KEGG metabolic pathways (octagons), biological functional classes (circles) and gene ontology terms (triangles) whereas others (black circles filled in with letters 'UK') did not display any enrichment. Black edges with ellipsoid target edges represent relationships with dual regulation (some of the transcriptional regulatory relationships within the sub-network are characterized by positive (activational) expression patterns and others by inverted (repressive) expression patterns between the regulator and the target gene). The meanings of all the other arrow symbols are as described in the legend of Figure 2.

metabolism respectively were also found to be downregulated. Sugars serve as substrates for the synthesis of glucans and associated by-products required for adherence to the tooth surface, biofilm formation as well as for the formation of organic acids which aid cariogenic disease progression [56-60]. The sub-network co-regulated by CodY (Additional file 8) meanwhile was found to be enriched with genes belonging to the metabolism and biosynthesis of amino acids, particularly the branched chain ones such as leucine, valine and isoleucine. The control of the metabolism of these amino acids by CodY has already been reported [38].
Hence, from the preliminary analysis of the regulatory response network, it can be stated that carolacton has an inhibitory effect on the central metabolism of sugars and amino acids by downregulating genes co-regulated by the global transcriptional factors CcpA and CodY.

\section{Immediate induction of the pyrimidine metabolism}

Peptidoglycan is an important constituent of the gram positive cell-wall. It is expected that cell membrane damage and biofilm inhibition would have a substantial effect on pathways and genes related to cell wall synthesis 
and metabolism. UDP-N-acetylglucosamine, a key intermediate in the biosynthetic process of the cell wall component peptidoglycan, is produced by glycolysis, sugar metabolism as well as the pyrimidine metabolic pathway [61]. Expression data indicate the absence of immediate modulation and at later time points the downregulation of the glycolytic pathway as well as of the pathways related to the metabolism of various sugars such as fructose, mannose and galactose. On the contrary, two pyrimidine biosynthesis gene clusters (namely the pyrEFDZ and pyrRPBA-carB operons) belonging to the PyrR sub-network and coding for the enzymes of the pyrimidine metabolism pathway (Figure 4) were upregulated by about 1 to $1.8 \log 2$-fold at $5 \mathrm{~min}$ post treatment (Figure $5 \mathrm{~A}$ ). It is of note that most of the genes in the pyrimidine metabolism pathway were not transcriptionally altered with the exception of the two strongly upregulated pyrEFDZ and pyrRPBA-carB operons. These operons encode enzymes catalyzing the biochemical steps leading to the production of UMP and UDP (see Figure 4) suggesting that this part of the pathway is specifically activated. An upregulation of the pyrimidine metabolism pathway would produce pools of UDP-N-acetlyglucosamine (UDP-N-AG) for peptidoglycan synthesis compensating carolactoninduced membrane and cell wall damage. The upregulation of pyrimidine biosynthetic steps was also observed in an S.aureus strain harboring a mutation of a two component system essential for cell wall metabolism [62].

\section{Carolacton affects glutamine metabolism}

Genes in the TRRN predicted to be co-regulated by the glutamine synthetase repressor $G \ln R$ along with $g \ln R$ itself were associated with a sharp downregulation initially (Figure 5B). It is known that glutamine is an important source of nitrogen for S. mutans [63]. Some of the GlnR-modulated genes in response to carolacton treatment included a glutamine transporter gene-cluster (SMU_1519-SMU_1522), the glutamine/glutamate biosynthesis operon $(g \ln R A-g l t A D)$, as well as an operon consisting of the putative ammonium transporter coding gene $n r g A$ and the nitrogen regulatory protein coding gene $\operatorname{gn} B$. Other glutamate and glutamine metabolism related genes such as citZH and idh [64], whose modulation was also reported to be mediated by $G \operatorname{lnR}$ [49], were downregulated initially until $20 \mathrm{~min}$ post carolacton addition. The protein products of the cit $\mathrm{ZH}$ and $i d h$

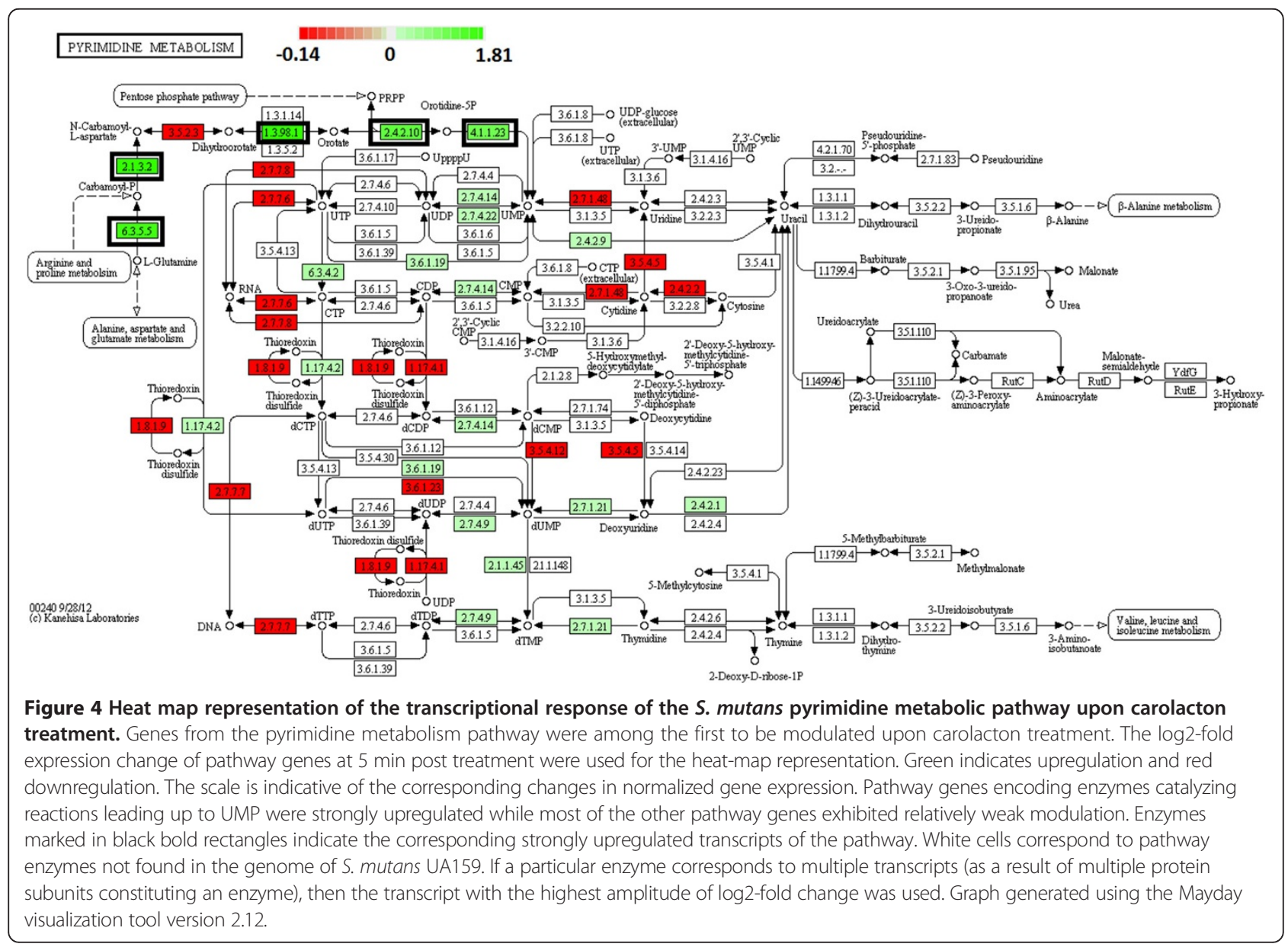




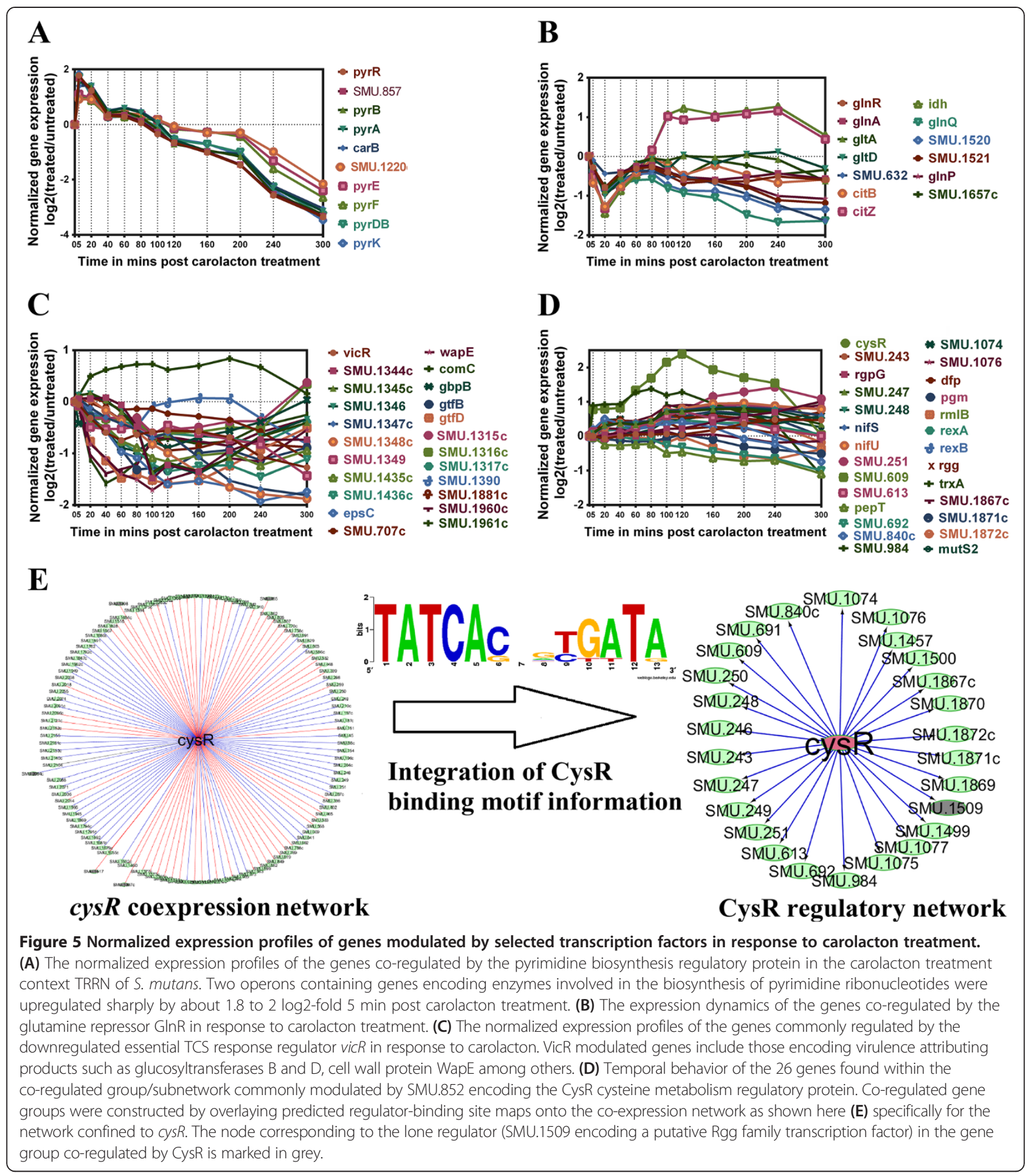

genes catalyze the steps leading to the formation of alpha-ketoglutarate from oxaloacetate (feeding the denovo glutamine synthesis pathway) [64]. The expression pattern of the genes predicted to be controlled by GlnR suggests an activational (positive) rather than an inverted (negative) relationship. But GlnR is known to function as a repressor protein. However, this does not seem to be a conflicting observation since $\mathrm{GlnR}$ orthologs have been known to have dual activator-repressor functions [65]. Hence, the S. mutans GlnR could also be a dual 
regulator given that several transcriptional regulators with dual functions have been reported earlier in $S$. mutans [66-68].

Glutamate is known to play a role in the acid tolerance response (ATR) mechanism of S. mutans [69-71] which is crucial for the survival and adaptation of $S$. mutans. Carolacton has the maximum effect in terms of membrane damage and cell death in actively growing biofilm cultures at low $\mathrm{pH}$. But the transcriptional changes (immediate downregulation until 20 min post treatment) of $g \ln R$ and its target genes occurred at a neutral $\mathrm{pH}$ (data not shown) thus ruling out ATR as a cause for the intial modulation of GlnR mediated genes. At later time points which correspond to a low $\mathrm{pH}$, the upregulation of the GlnR-modulated genes occurred although a downregulation should have been the expected trend to enhance the ATR. This suggests that carolacton directly or indirectly diminishes the ATR thereafter. Alternatively, the upregulation of the glutamine metabolism genes comprising the GlnR co-regulated gene group after the initial downregulation could possibly be explained as a means to provide precursors for peptidoglycan biosynthesis. Thus, it is plausible that the pyrimidine metabolism is induced to feed the peptidoglycan biosynthesis during the initial phase (until 20 min after carolacton treatment) followed by the upregulation of the glutamine metabolism related genes during the later phase.

\section{TRRN genes co-regulated by the essential response regulator VicR}

The microarray data show that vicKR, one of the earliest responding TCSs upon carolacton treatment, was downregulated. The vicKR TCS plays a major role in the biofilm formation, competence development, oxidative stress tolerance, acid tolerance, autolysin production, glucan and fructan metabolism of $S$. mutans [27,72-77]. In addition, the response regulator $v i c R$ has also been identified as being essential for the survival of S. mutans, i.e. it is an essential gene which cannot be deleted [8]. The gene group co-regulated by VicR (Figure $5 \mathrm{C}$ ) in the TRRN consisted mostly of downregulated genes including those encoding surface structure and cell wall proteins such as glucosyltransferases (gtf) $\mathrm{B}$ and $\mathrm{D}$, glucan binding protein $\mathrm{B}$ (gbpB), and the sortase-anchored cell wall protein wapE [78] among others. This is consistent with the previous study [5] whereby all the genes including the ones described here and co-expressed with the downregulated $v i c R$ had reduced transcript abundances. The evidence put together suggests that carolacton could interfere with the signalling mechanisms related to the activity of this essential response regulator thereby exerting its biofilm inhibitory and membrane damaging effects. Some of the genes such as $g t f B, g b p B$ and $\operatorname{com} C$ in the VicR coregulated gene cluster were already known to be direct transcriptional regulatory targets $[76,79,80]$ of the VicR protein thus contributing to an independent verification of our network prediction based on time-series microarray data and binding motif information.

\section{Modulation of genes co-regulated by the cysteine metabolism controlling transcription factor CysR}

Genes connected by edges to $c y s R$ (encoding a regulatory protein controlling the metabolism of sulfur containing amino acids [81]) in the co-expression network had both inverted and positive expression patterns compared to that of cysR (Additional file 2). After combining the CysR binding motif information [81], directionality was assigned to the edges after which all of the cysR co-regulated genes were having only positive expression patterns (Figure 5D) in relation to cysR. CysR was found to modulate 26 TRRN genes (Figure 5E) making it the transcriptional regulator with the second highest degree (number of modulated genes) in the carolacton context TRRN. Some of the genes transcriptionally modulated by CysR include SMU_609 encoding a putative-40 K cell wall protein precursor, SMU_246 encoding a putative glycosyltransferase- $\mathrm{N}$-acetylglucosaminyltransferase and SMU_984 whose gene product has been predicted to be an uncharacterized autolysin among others. Besides, SMU_1509 whose gene product is a Rgg family [82] regulator protein was also observed to be among the CysR sub-network as were genes encoding putative Nif proteins related to nitrogen metabolism and those such as the SMU_1074-SMU_1077 operon involved in cysteine metabolism. Statistically significant enrichment for various functional categories was not observed within the CysR sub-network and this might be attributed to the low number of genes from any given category. Nevertheless, the functional relevance of the individual genes (such as SMU_609 which is one of the strongest modulated genes with $>2 \log 2$-fold upregulation in response to carolacton treatment) within the CysR sub-network as well as its connectivity in the TRRN point out the importance of CysR in the response of $S$. mutans biofilms to carolacton.

\section{Experimental verification of the predicted regulatory interactions between MbrC (BceR) and the murMN-SMU_718 operon}

By combining gene expression and binding motif information, $m b r C$ was predicted to regulate four so far unidentified target genes (the SMU_716-SMU_717-SMU_718c operon and SMU_610 encoding the cell surface antigen SpaP) in the carolacton treatment context TRRN. The autoregulatory response regulator $\mathrm{MbrC}$-also known as BceR- is encoded within a four-gene operon ( $m b r A B C D /$ bceABRS) and has been shown to regulate cell envelope stress response mechanisms in S. mutans [50]. Ouyang et al. demonstrated that $\mathrm{MbrC}$ transcriptionally regulates 
the genes SMU_302, SMU_862, SMU_1006 and SMU_1856 by binding to their promoter elements via a conserved binding motif. The binding motif consensus consists of a conserved pair of inverted repeats separated by 2 variable nucleotides (TTACAAnnTTGTAA) [50].

Among our predicted MbrC target genes, SMU_718c codes for a hypothetical protein with a haloacid dehalogenase-like domain, and SMU_716 and SMU_717 encode two different enzymes, MurN and MurM respectively. These enzymes catalyze the last steps of the peptidoglycan biosynthesis pathway and also play an important role in imparting resistance to cell wall-acting antibiotics [83-86]. Figures $6 \mathrm{~A}$ and $\mathrm{B}$ illustrate the coexpression of $\mathrm{mbrC}$ with the murMN-SMU_718c operon genes and the presence of the potential MbrC binding site (TTACAA-AT-TTCTAC) upstream of the putative target murMN-SMU_718c operon respectively. This potential binding site differs from the motif consensus identified by Ouyang et al. [50] by the presence of two substitutions in the inverted repeat and is located upstream (between -33 and -20 ) of the transcriptional start site of the murMN-SMU_718c operon.

To verify this predicted regulatory relationship, Electro Mobility Shift Assays (EMSA) were carried out. The promoter region of SMU_1006 served as a positive control as the binding of $\mathrm{MbrC}$ to it was reported previously [50]. An unrelated DNA fragment (around 100-150 bp, lower DNA band in all the lanes) was added in all the samples to prove the specificity of the binding reaction. As shown in Figure 6C, the results confirmed the binding of $\mathrm{MbrC}$ to the SMU_1006 promoter as well as the necessity of the consensus motif for the binding of $\mathrm{MbrC}$ to its target. When an unrelated transcriptional regulator VicR was used instead of $\mathrm{MbrC}$ in the binding reaction as a control to test the specificity of the assay, no shifting of the DNA band was found while with increasing MbrC concentrations, a clear shift was observed (upper band around
A

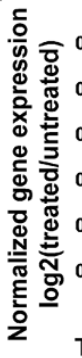

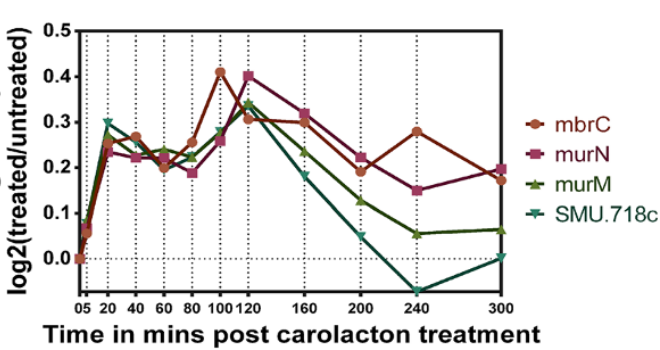

\section{B}

GENE

UPSTREAM REGULATORY SEQUENCE WITH THE SPACED DYAD MbrC BINDING MOTIF

POSITION STRAND

SMU 1006

SMU 302

SMU 1856

SMU 762

SMU $718 \mathrm{C}$
TCAATGCTTACAATTTTGTAAGCTACGATTCTTTAAGT $-59 \quad$ D TGAGGACTTACAAAGGTGTAAGTTCTATCTTGATTTTT -53 D GGTTIATTTACAAAATTGTAAGTTATAGTACAAAGACTG -40 D TTAAAACTTACAATTTTGTAAGTTTIAACTTTAATAATG -19 D TITCTAATTACAAATTTCTACAGACTATCTIACTAAAAA -33 R

\section{C}

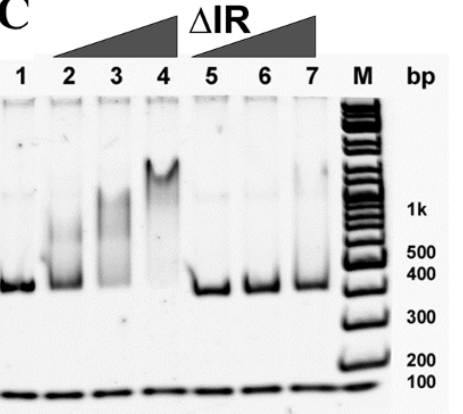

Lane $M$ : Ladder

Lane 1 ( control) : 20 pmol VicR protein added to the intact SMU_1006 promoter

Lane $2: 5 \mathrm{pmol} \mathrm{MbrC}$ protein added to intact SMU_1006 promoter

Lane $3: 10 \mathrm{pmol} \mathrm{MbrC}$ protein added to intact SMU 1006 promoter

Lane 4 : $20 \mathrm{pmol} \mathrm{MbrC}$ protein added to intact SMU_1006 promoter

Lane $5: 5 \mathrm{pmol} \mathrm{MbrC}$ protein added to intact SMU_1006 promoter with deleted MbrC binding motif

Lane $6: 10 \mathrm{pmol} \mathrm{MbrC}$ protein added to intact SMU_1006 promoter with deleted MbrC binding motif

Lane $7: 20 \mathrm{pmol} \mathrm{MbrC}$ protein added to intact SMU_1006 promoter with deleted MbrC

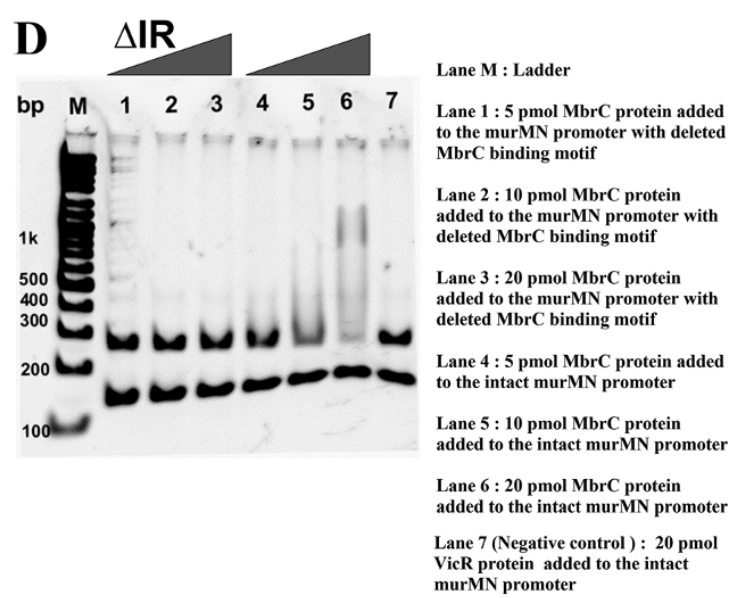

Figure 6 Experimental verification of the predicted transcriptional regulation of the murMN-SMU_718c operon by the response regulator MbrC. The predicted transcriptional regulatory relationship was based on a well correlated (A) expression profile between $m b r C$ and the murMN-SMU_718 operon as well as the presence of a (B) putative MbrC binding site (TTACAA-AT-TTCTAC) in the upstream regulatory regions of the murMN-SMU_718 operon. The alignment among the MbrC binding sites in other experimentally verified targets (black) reported by Ouyang et al. [50] and the putative site upstream of the predicted target (red) murMN-SMU_718 operon is shown. The signature repeats of the MbrC binding motif are italicized, underlined and shown in bold. (C) Binding of MbrC to the promoter region of the gene SMU_1006 (positive control) was verified using Electro Mobility Shift Assays (EMSA), as already reported by Ouyang et al. [50]. (D) EMSA also provided the verification of the in-vitro binding of the MbrC protein to the promoter region of the predicted target murMN-SMU_718c operon via the putative binding site thus confirming that the latter is a transcriptional regulatory target of $\mathrm{MbrC}$. The triangles indicate increasing concentrations of $\mathrm{MbrC}$ in the binding reactions. Black triangles followed by IR indicate target DNA fragments lacking the MbrC binding site. 
$350 \mathrm{bp})$. No significant shift was observed upon deleting the binding motif from the SMU_1006 promoter. Similarly, $\mathrm{MbrC}$ also bound to the promoter region of SMU_718c as indicated by a band shift at MbrC concentrations higher than or equal to $5 \mathrm{pmol}$ (Figure 6D). With increasing amounts of $\mathrm{MbrC}$ (5-20 pmol) present in the binding reaction mixture, a shift from 250 bp (upper DNA band) to around $1 \mathrm{kbp}$ was observed. No shift was observed in the control lane corresponding to the reaction mixture containing an unrelated transcriptional regulator VicR (whose binding site was not present in the murMNSMU_718 promoter) confirming the absence of nonspecific binding. Furthermore, the deletion of the $\mathrm{MbrC}$ binding site from the murMN-SMU_718 promoter also abolished the binding of MbrC to its DNA target. Thus the observations from the EMSA studies have confirmed that the murMN-SMU_718 operon is a direct target of the $\mathrm{MbrC}$ response regulator as predicted from the regulatory response network. The two substitutions present in the MbrC binding site upstream of the murMN-SMU_718c operon could reflect a less stringent binding of $\mathrm{MbrC}$. Accordingly, the shift of the SMU_718c target DNA occurred at a higher $\mathrm{MbrC}$ protein concentration (10 pmol) than that $(5 \mathrm{pmol})$ observed for the corresponding SMU_1006 target DNA.

Our finding that $\mathrm{MbrC}$ directly controls the expression of two enzymes involved in a central step of cell wall synthesis further highlights the importance of this response regulator for the cell envelope stress response and for maintaining cell wall homeostasis. The reconstitution of the Gram positive bacterial cell wall during growth is intricately linked to the synthesis of peptidoglycan polymer chains [87]. Both murM and murN encode alanine adding enzymes involved in the branched peptide peptidoglycan biosynthetic pathway and have also been reported to be primary determinants of the peptidoglycan stem peptide structure $[83,86]$ which in turn affects various virulence properties such as antibiotic resistance [88-90] and pathogenesis [91]. Thus, although the transcriptional induction of the murMN-SMU_718 operon and the mbrC gene is relatively weak following carolacton treatment, it might still be significant since only a few copies of the enzymes might potentially be required to catalyze the peptidoglycan biosynthetic reactions. Post transcriptional regulation of enzymes involved in cell wall synthesis and cell division might also significantly contribute to a quick and efficient adaptation of the cell to maintain cell wall homeostasis. Thus small transcriptional changes might result in strong changes of enzyme activity. Induction of the $\mathrm{MbrC}$ regulon by carolacton is reminiscent of the previously observed [50] bacitracin induced cell envelope stress response mediated by this regulon in S. mutans. This finding further points out the influence of carolacton on cell wall synthesis as reported previously [5].

\section{Deletion mutants of "key" transcriptional regulators}

Based on the inferred TRRN and co-expression networks, single gene deletion mutants corresponding to five different transcriptional regulators (CysR, Rgg, GlnR, SpxA, and FabT) were constructed. We chose these potential key genes for experimental evaluation based on the following criteria. Next to that of CodY, CysR (SMU_852) was identified as the regulator with the highest connectivity in the regulatory response network. CodY is a well-known global master regulator [38] and its deletion has been shown to have obvious phenotypic effects such as the reduced capacity to form biofilms [38]. Since biofilm growth is a prerequisite for the inhibitory action and membrane damage caused by carolacton, $\operatorname{cod} Y$ was not chosen as a deletion candidate.rgg (SMU_1509) was identified as the only transcriptional regulator encoding gene among the CysR coregulated genes and hence was chosen as a candidate for deletion. Alterations in the cell wall metabolism could play a critical role in the response of $S$. mutans biofilms to carolacton due to the observed membrane and cell wall damage [3,5]. GlnR was chosen as a possible knock-out target due to its importance in peptidoglycan biosynthesis and cell wall metabolism via many of the glutaminemetabolism and transport genes which it modulates as observed in the TRRN. spxA (SMU_1142) and fabT (SMU_1745) were identified as the top two nodes purely based on connectivity in the co-expression network and hence chosen for further testing. The other genes from the co-expression network were not chosen in this work.

The susceptibility to carolacton treatment of $20 \mathrm{~h}$-old static biofilms of the corresponding key regulator gene deletion mutants was tested using Live/Dead viability staining. As shown in Figure 7A, carolacton only marginally reduced the viability ( $\sim 5-10 \%$ inhibition) of the biofilms of the $c y s R$ gene deletion strain, while complementation of cys $R$ in trans fully restored the carolacton sensitive phenotype of the wildtype (approximately $45-55 \%$ inhibition of viability). It was previously shown that carolacton exclusively damages growing biofilms [5]. The observed strong loss in sensitivity of the cysR gene deletion strain to carolacton was not biased due to poor or significantly slower growing mutant biofilms since the final growth yield and doubling time of the cysR gene deletion mutant was only slightly reduced in comparison to the wildtype (data not shown). Strikingly, the $s p x A$ gene deletion strain displayed impaired ability to grow under acidic conditions [92], a condition known to be essential for the carolacton induced membrane damage in S. mutans [5]. However, all the tested strains with the $f a b T$ or $c y s R$ deletion showed similar susceptibilities to carolacton relative to the wildtype (Figure 7A). For the fabT deletion mutant though, no final conclusion regarding its sensitivity to carolacton treatment could be drawn since the mutant strain grew very poorly under the tested conditions and formed very 


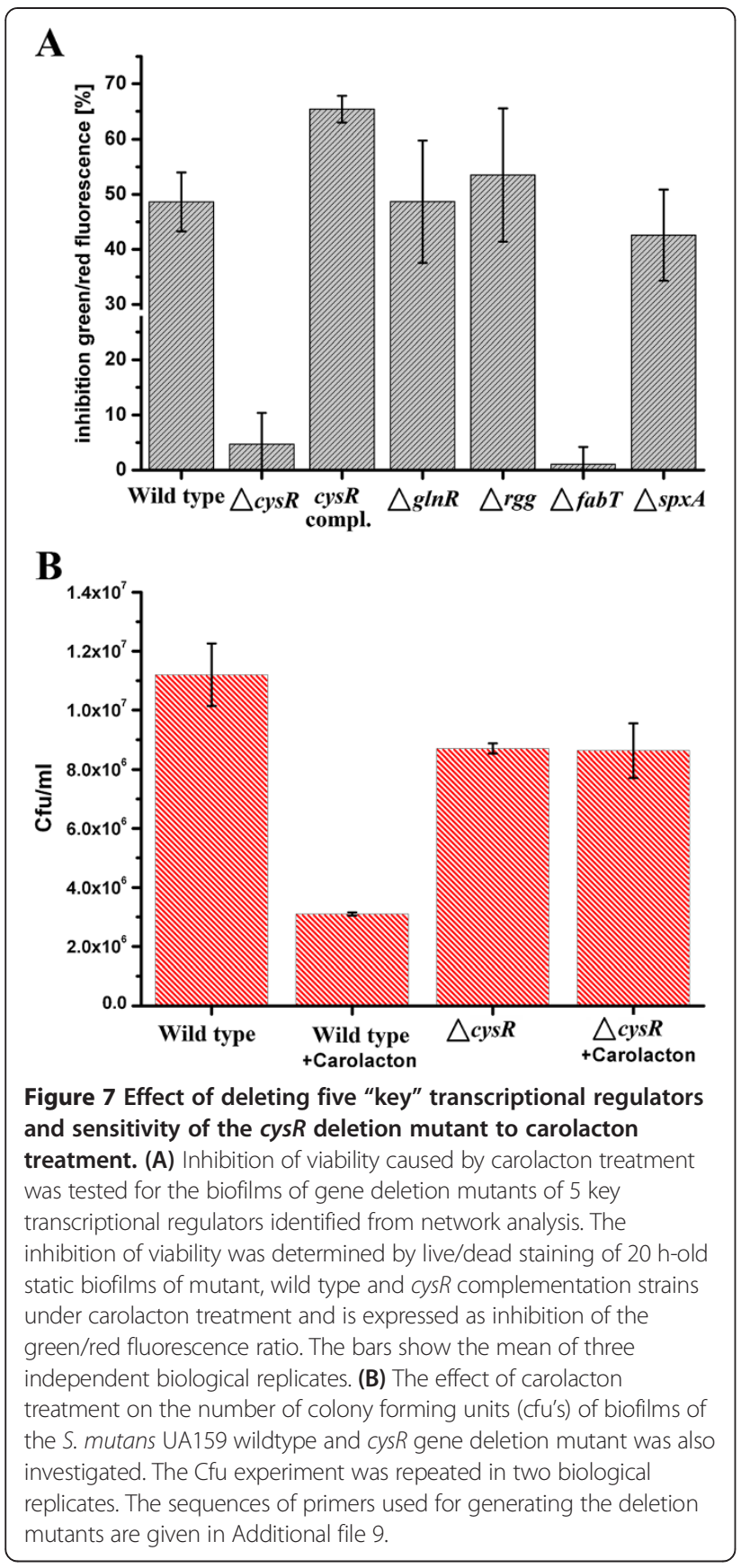

thin biofilms. Since growth is a prerequisite for the membrane damage caused by carolacton treatment [5], the results of the Live/Dead staining for the fabT mutant should be interpreted with caution. However, the membrane integrity of the fabT deletion mutant was highly compromised (data not shown), as determined by live/dead staining. The importance of $f a b T$ for maintaining membrane integrity in $S$. mutans biofilms might also be indicative of its involvement in the membrane damage caused by carolacton treatment. Furthermore, $f a b T$ was identified as a top structural hub of the inferred co-expression network, indicating its essential physiological role, and the deletion of which is therefore expected to have a high chance to cause poor growth.

\section{The role of cysR in the response of $S$. mutans biofilms to carolacton}

From our initial susceptibility studies of mutant biofilms under carolacton treatment, cys $R$ could be identified as being essential for the sensitivity of growing $S$. mutans biofilms to carolacton treatment. The almost complete loss of sensitivity of the cysR mutant to carolacton treatment was independently confirmed by the determination of colony forming units of carolacton treated and untreated biofilms of the wild type and cysR gene deletion strains. While the wildtype showed a reduction of CFUs of approximately $75 \%$, the carolacton treated and untreated mutant cells showed almost no difference in the amount of colony forming units (Figure 7B). Thus the experiment clearly confirms the essential role of $c y s R$ in the response of $S$. mutans biofilms to carolacton treatment, as predicted from the TRRN. The genome of S. mutans encodes 4 LysR-type transcriptional regulators, of which 3 (CysR, MetR, HomR) are phylogenetically linked and control the supply of sulfur amino acids $[81,93]$. CysR is known to modulate the expression of genes involved in cysteine metabolism in S. mutans [81]. Here, we provide data showing that apart from its role in cysteine metabolism, CysR is essential for the response of $S$. mutans biofilms to the biofilm inhibitor carolacton. This implies a potential role of CysR in cell wall maintenance, as this trait was shown to be impaired in carolacton treated biofilm cells and cell wall changes are most likely responsible for cell death caused under acidic conditions [5].

The inferred transcriptional regulatory targets of CysR, as identified after the integration of contextual coexpression correlation and binding motif information, indeed comprise genes involved in maintaining cell wall homeostasis. Genes SMU_984 and SMU_609 encode two autolysins which were not characterised so far. Interestingly, both genes were immediately ( 5 min after treatment) and constantly upregulated after carolacton treatment and belong to the strongest regulated genes of our time-resolved transcriptome study. SMU_609 encodes a $40 \mathrm{~K}$ cell wall protein which was shown to exhibit murein hydrolase activity using a peptidoglycan zymogram assay and is likely linked to the cell surface via the sortase system $[78,94]$. As cell wall synthesis during growth depends on a balanced interplay between build-up and breakdown of the cell-wall, enhanced autolysin activity might promote cell lysis [95] and thus explain the influence of CysR on cell death. However, deletion of SMU_609 and SMU_984 did not cause a carolacton insensitive phenotype (data not shown). Another CysR regulated gene, rgpG (SMU_246), encodes 
a putative UDP-N-acetylglucosamine undecaprenyl-phosphate GlcNac 1-phosphate transferase. This enzyme catalyses the first membrane localized step of the biosynthesis of various polymers of the bacterial cell wall. The other potential target genes include the gene cluster SMU_1074-SMU_1077 which encode putative metabolic enzymes belonging to the pathways related to cysteine metabolism and is concurrent with the known role of cysR in cysteine metabolism [81].

It should also be noted that cysR and its paralog cps $Y$ are located in close vicinity to the genes of the de novo pyrimidine biosynthesis pathway on the genome of S. mutans. The pyrimidine biosynthesis genes were shown to be the strongest upregulated genes $5 \mathrm{~min}$ post carolacton treatment. The functionality of pyrimidine metabolism, especially of the pyr gene clusters upregulated specifically under carolacton treatment, could possibly be linked to the availability and synthesis of cell wall precursors. In prokaryotes, genes encoding products involved in the same or related biological functions are often located in close neighbourhoods on the genome [96-98].

Moreover, for the S. iniae cysR ortholog cps $Y$, it was shown that this LysR type transcriptional regulator (LTTR) induced cell wall changes essential for the intracellular survival of this invasive pathogen in neutrophils [99]. Interestingly, in the study of Allen et al. [99], it was determined that deletion of cps $Y$ alters the cell surface charge, muropeptide composition and susceptibility to lysozyme treatment. This is fully in accordance with the current understanding of the mode of action of carolacton [5]. However further experiments are necessary to verify the targets of CysR experimentally (e.g. EMSA experiments with purified CysR protein) and to fully address the role of cysR in cell wall metabolism e.g. by knocking out the CysR regulated autolysins. Altogether, our data strongly suggest a so far unidentified role of the CysR transcriptional regulator in cell wall homeostasis.

A highly interesting aspect of the network prediction is the finding that CysR potentially regulates another transcriptional regulator (Rgg). As the carolacton specific regulatory response network contains albeit only two levels of hierarchy, CysR can thus be considered as a potential global regulator. Rgg transcriptional regulators associated with small hydrophobic peptides (SHP) were identified to represent a part of a novel quorum sensing mechanism in Streptococci [82,100]. Rgg proteins have been identified and conserved in nearly all streptococci [101]. This mechanism works independently of a TCS, but senses the signalling molecule inside the cell after its internalisation via an oligopeptide permease [82]. But the Rgg knockout strain showed comparable susceptibility to carolacton treatment as the wildtype strain (Figure 7A) thus excluding the possibility of Rgg being a critical regulator mediating the observed effects of carolacton.
With respect to cysR however, its deletion mutant displayed a loss of sensitivity to carolacton as a result of a reduction in the inhibition of viability caused by carolacton. Nevertheless, CysR might not represent the primary target of carolacton as the treated biofilm cells of the cysR gene deletion mutant still exhibit an inhibition of about $10 \%$. Moreover, phenotypic differences between carolacton treated and untreated cys $R$ mutant biofilm cells were observed, indicating that carolacton can still bind to its target(s) and induce changes in the cellular morphology. Nevertheless, the lethal effects of carolacton treatment apparently rely to a large extent on the presence of an intact copy of the cysR gene. Another interesting question is the link of CysR to the PknB controlled regulon, as $p k n B$ was also shown to be essential for carolacton mediated cell death [5]. With PknB being a global regulator of cell division [102] and virulence attributes [103], the regulons of PknB and CysR might converge at the level of the modulated autolysins. The CysR coregulated genes SMU_984 and SMU_609 were 2 of the 3 strongest modulated genes in the transcriptome analysis of an exponentially growing $p k n B$ deletion strain [103]. However, as the deletion mutants of SMU_609 and SMU_984 are still susceptible to carolacton treatment, this potential overlap of the regulatory systems does not provide an explanation why both the $p k n B$ and $c y s R$ deletion mutant biofilms exhibited almost no sensitivity to carolacton treatment.

Since LTTRs show a high degree of sequence conservation, control important metabolic pathways of sulfur containing amino acids, and since sulfur is essential for the active sites of many enzymes, the transcriptional regulator CysR might also be an attractive drug target. Co-factor binding is required for its transcriptional activation, and the likely cofactor could be O-Acetylserine (OAS) [81]. Thus, small molecule inhibitors could potentially be designed to compete with the co-effector molecule for the binding site on this LTTR and to finally block its biological function. To conclude, we have shown here strong evidence that the role of CysR may be much more diverse and important than previously expected. Traits essential for survival like cell wall metabolism and sulfur amino acid supply are controlled by this LTTR. Thus CysR might represent an attractive novel streptococcal drug target.

\section{Linkage between the PknB and CysR regulons}

Deletion of $c y s R$ almost completely prevents cell death in carolacton treated biofilms, a finding previously reported for the gene deletion strain of the Serine Threonine Protein Kinase (STPK) encoding gene $p k n B$ as well [5]. This instantly raises the question whether the regulons of these two proteins overlap or if $\mathrm{PknB}$ is located upstream of CysR in the regulatory cascade and thus controls the latter's activity. Both regulators putatively modulate the 
expression of the two carolacton responsive autolysins SMU_984 and SMU_609, which might explain the shared insensitive phenotype to carolacton of their mutant strains.

However, in this study, we focus only on the transcriptional network analysis, while PknB modulates its target genes via protein phosphorylation at the post-transcriptional level $[9,103,104]$. Thus the question whether and on which level the modulated networks of PknB and CysR converge and if they represent the essential pathway for the carolacton mediated cell death cannot be fully answered from the transcriptional regulatory network analysis. To quantitatively understand the effects of carolacton on cell metabolism and to determine the missing layers in the regulatory cascades of CysR and PknB, it is necessary to consider the regulatory effects of small noncoding RNAs [105] and other post-transcriptional and post-translational modifications [106,107] including modulation by other signalling pathways [108]. Transcriptional network analysis is generally lacking in information about these important regulatory mechanisms which have been shown to be essential for cellular metabolism and homeostasis $[105,106,109]$. Nevertheless, we have demonstrated here the high merit of transcriptional network prediction and analysis to identify a key regulator mediating cell death in S. mutans biofilms in response to the biofilm inhibitor carolacton.

\section{Conclusion}

We have used a combination of transcriptional network prediction and experimental verification to analyse the response of $S$. mutans biofilms upon treatment with the biofilm inhibitor carolacton. According to our knowledge, this is the first study inferring a genomewide transcriptional regulatory response network for S. mutans using heterogeneous data including a timeseries transcriptomic dataset and transcription factor binding site information. Based on the inferences from the regulatory network, we experimentally verified important predicted transcriptional regulatory interactions between the cell envelope stress modulating TCS response regulator $\mathrm{MbrC}$ and the operon harbouring the murMN genes encoding cell wall metabolism associated enzymes. CysR, a regulator known to modulate cysteine metabolism, was predicted and experimentally verified to be an essential key regulator for the mode of action of the biofilm inhibitor carolacton. Our data strongly suggest a role of $c y s R$ in cell wall metabolism, cell division and cell surface biogenesis, apart from its already known role in cysteine metabolism and sulfur supply in S. mutans, thus highlighting its potential as an attractive novel streptococcal drug target. We here also demonstrate the high predictive power of the network construction and analysis strategy used in this work.

\section{Methods}

\section{Transcriptomics}

Static biofilm cultures of S. mutans UA159 were grown in routine 24 well plates (Greiner Bio One, Frickenhausen, Germany) using $800 \mu \mathrm{l}$ Todd Hewitt Broth (Becton Dickinson, Heidelberg, Germany) supplemented with $0.5 \%$ sucrose (Sigma, Taufkirchen, Germany) per well as media. Overnight cultures of planktonic cells in Todd Hewitt Broth were diluted in the biofilm medium to a starting OD of 0.05 and biofilms were grown for 3 hours under anaerobic conditions $\left(80 \% \mathrm{~N}_{2}, 10 \% \mathrm{CO}_{2}, 10 \% \mathrm{H}_{2}\right)$ in a chamber (Don Whitley Scientific, Shipley, England). Thereafter, the supernatants were completely removed and exchanged with fresh medium (control samples) or either fresh medium supplemented with $2.5 \mu \mathrm{g} / \mathrm{ml}$ carolacton (treated samples). For the control, an equivalent volume of methanol was added. Samples were taken after $0,5,20,40,60,80,100,120,160,200,240$ and 300 minutes by removing the supernatant and adding $200 \mu \mathrm{l}$ fresh medium and $400 \mu \mathrm{l}$ of RNA protect (Qiagen, Hilden Germany). Biofilms representing one condition were scraped from at least 3 individual wells and pooled together. Cells were pelleted by centrifugation, washed with Molecular biology grade water (Sigma, Taufkirchen, Germany) and lysed with a combination of Lysozyme/ mutanolysin treatment and mechanical disruption of cells with glass beads [5]. Total RNA was extracted using the RNeasy Kit (Qiagen, Hilden Germany) as recommended by the manufacturer. The overall experiment was repeated once.

$2 \mu \mathrm{g}$ of total RNA from each sample was labeled with Cy3 and Cy5-ULS (Kreatech, Netherlands) according to the protocol of the company. Fragmentation of RNA and further processing was performed according to the Agilent hybridization kit procedure. Samples were hybridized in a dye swap design on $8 \times 15 \mathrm{~K}$ S. mutans custom arrays (Agilent, Böblingen, Germany) for $14 \mathrm{~h}$ at $65^{\circ} \mathrm{C}$ as previously described [5].

The array design is described in detail in [5]. Slides were washed and scanned as recommended by Agilent. For feature extraction, the Agilent extraction software (V. 10.7) was used. The further data analysis was performed using the $\mathrm{R}$ package LIMMA. For dye specific within-array normalization, the LOWESS algorithm was applied. Quantile normalization was used for between arraynormalization. Genes corresponding to rows with blanks were omitted. From the log transformed and background corrected median signal intensities, the fold change between treated and untreated samples was calculated for every gene at each time point. The $\log 2$ (treated/ untreated) expression ratios were then subjected to a within-gene normalization. Genes with a log2-fold ratio greater than 0.8 were regarded as genes corresponding to transcripts with altered abundances (GTAAs). 


\section{Functional context enrichment analysis}

Enrichment analysis was performed separately for the upand downregulated GTAAs (at every measured time point) with respect to each of the known biological functional classes and KEGG metabolic pathways in order to gain an overview of the various functional categories affected by carolacton treatment. 15 main and 97 sub functional classes of $S$. mutans UA159 were compiled using the gene annotation information furnished in the Oralgen database (version 2011 and now re-named as The Bioinformatic Resource for Oral Pathogens). The gene membership information of S. mutans UA159 corresponding to 84 different pathways was retrieved from the KEGG database (version 2011). The hypergeometric test with Benjamini-Hochberg correction was used to quantify the significance of the overlap between the GTAAs and the functional categories. A corresponding significance score was also calculated as a negative logarithmic function of the corrected $\mathrm{P}$ value. The significance score is a user friendly measure of the relevance of the overlap between functional categories and the GTAAs. The higher the significance score, the more relevant is the overlap. Categories with significance score greater than 0 represented significant enrichment.

\section{Inferring gene-gene correlation relationships from transcriptomic data using the Trend Correlation (TC) method}

The TC method [32] allows the inference of gene-to-gene time-lagged positive or negative "associations" or pairs of genes based on extracting the main features of the change trend and the correlation of gene expression changes between consecutive time points [32]. Time lagged coexpression relationships are those in which the expression patterns of two genes are better correlated when a time shift is introduced [32]. In this work, we adapted the TC method by calculating the correlation coefficient from the change rates rather than change levels between consecutive time points since the time interval of the measurements was not constant (personal communication, Feng $\mathrm{He}$ ). Potential co-expression relationships were checked for every possible regulator-gene pair given the minimum number of expression measurements in the chosen time window was four. The genetic response network consisted of optimal expression relationships which started either at 0,5 or 20 min post carolacton treatment in order to capture the correlations mediating the initial response to carolacton. The significance of correlation of a gene-pair for every time-window is measured by the co-expression P-value by procedures described previously in the TC method by $\mathrm{He}$ et al. [32]. The individual P-values of correlation for a gene pair within every considered timewindow are compared among each other. Optimal correlation for a gene pair refers to the time window within which the correlation between the genes is most significant statistically. Parameters such as the correlation coefficient, the trend score, and the P-value were calculated as described previously [32] to characterize the degree and randomness of correlation between the temporal expression patterns of genes. The association between two genes was considered to be statistically significant if the corresponding P-value of their co-expression was determined to be lower than the optimal P-value cutoff calculated using standard performance metrics as explained below.

\section{Performance metrics for the determination of the optimal P-value threshold}

The optimality of the P-value threshold was determined based on the F-score (Eq. 1) which in turn is a function of the equilibrium between the Recall (Eq. 2) and Precision (Eq. 3) functions which are defined below.

- F-score: this is predictive of the balancing property or equilibrium between the Precision and Recall parameters. The F-score usually reaches a maximum peak at the P-value optimum.

F-score $=\frac{2 * \text { Precision } * \text { Recall }}{\text { Precision }+ \text { Recall }}$

- Recall (also termed as True Positive Rate or Sensitivity): defined as the fraction of true "associations" which are inferred as significant by the TC method

Recall $=\frac{\text { Number of true positives }}{\text { Number of true positives }+ \text { Number of false negatives }}$

- Precision (also termed as Positive Predictive Value): defined as the fraction of inferred significant "associations" which are true

Precision $=\frac{\text { Number of true positives }}{\text { Number of true positives }+ \text { Number of false positives }}$

Gene pairs within the same operon because of their virtue of coherent co-expression were used as the "gold standard" or true positives. False positives constitute the inferred significant "associations" between genes from different operons whereas false negatives represent the intraoperonic gene pairs which were considered as insignificant and which were seemingly missed out at the particular P-value threshold. The operon information was predicted by Pathway Tools [110]. Differential edges $[111,112]$ in response to carolacton treatment were pooled together to form the final co-expression network. 
Construction of Position Specific Scoring Matrices (PSSMs) representing transcription factor binding motifs

Experimentally verified DNA binding sites of $S$. mutans transcription factors were retrieved from literature when available. The comparitive genomics based approach using the concept of regulogic extrapolation as explained in [33] was carried out to retrieve orthologous regulatory relationships in $S$. mutans from experimentally verified relationships in B. subtilis. Validated binding sites of B. subtilis transcription factors were retrieved from the DBTBS database [113]. OrthoMCL [114], which is a genome-scale algorithm for grouping matching protein sequences, was used to identify orthologous genes shared between $S$. mutans UA159 and other gram positive bacterial species including some representative species from the Streptococcus genus. The OrthoMCL results were verified by manually searching for the presence of commonly occurring domains in the protein sequences of the orthologous pairs of transcription factors. To increase the reliability of a particular predicted regulog in $S$. mutans, a check was performed to ascertain if the regulog was conserved in at least some species within the Streptococcus genus. Consensus motifs constructed from too few binding sites have a low predictive value [37]. Hence the available binding sites were enriched with corresponding putative sites from regulogs identified from different species within the Streptococcus genus. In addition to the PSSMs constructed from manually identified regulogs, PSSMs were also compiled from already assembled regulogic sites retrieved from the RegPrecise database [36] and from phylogenetic footprinting studies. Each resulting set of binding sites was submitted to consensus, which is a tool for mining out conserved motifs. The tab-formatted PSSMs were then converted into TRANSFAC formatted PSSMs which enable an user-friendly organization of pattern matching results. Additional file 3 lists the transcription factors with their corresponding binding site information.

\section{Prediction of putative binding sites by Pattern Matching}

Since the predictive capacity of each PSSM (due to its varying information content) differs from case to case, a generic or common P-value could not be used to screen out "putative-target hits" (i.e. genes or operons harboring binding sites predicted by the corresponding PSSMs). The RSAT tool termed matrix-quality [37] was used to objectively determine the optimal P-value for every PSSM. The TRANSFAC formatted PSSMs were then employed to search for putative sites using the tool matrix-scan (http:// www.rsat.eu/) [115,116] with the determined optimal P-values set as the cut-offs. Putative sites were searched for in the upstream regions (1000 bps upstream of the start codon with no overlap with the coding regions of the preceding ORF) of all genes in the S. mutans UA159 genome. A markov order of 0 and a background model comprising $S$. mutans specific upstream regions (no overlap with ORF coding regions) was used during the pattern matching scan. The resulting sites with a P-value smaller than the determined optimal P-value were considered as significant. In cases of PSSMs for which the optimal P-values could not be determined due to the inherent low complexity of the motifs [37], a whole genome stringbased site identification was performed using the nonmatrix based tool genome-scale dna pattern (http://www. rsat.eu/) [115] with an allowance for a maximum of 2 mismatches. Both the forward and reverse strands were subject to the pattern matching procedure.

\section{Analysis of sub-networks}

Even if the expression profiles of not all the genes in the same operon are well correlated with the expression of a certain regulator gene, we assume that all the genes in the same operon will be regulated by the regulator provided that the corresponding binding sites are found in the upstream regulatory regions of the operon. This is what we refer to as operon-oriented adjustment.

To obtain the TRRN, the co-expression network was superimposed onto the regulator-target gene binding site map. The genes in each co-regulated TRRN group or subnetwork after operon-oriented adjustment were analyzed to detect any significant over-representation of categories such as biological functional classes, KEGG metabolic pathways and gene ontology terms. The hypergeometric distribution was employed to determine over-represented categories with the network analysis tool compare-classes (http://www. rsat.eu/). Self and reciprocal comparisons were avoided. Enrichment events with significance scores $>=0$ were considered as statistically significant. Gene ontology information was retrieved from the Pathosystems Resource Integration Center (PATRIC) [117]. All the network visualizations were performed using Cytoscape [118]. Network motifs were analyzed using the tool MFINDER [119]. The significance of the discovered motifs in the real network was determined in comparison to their occurrences after 10000 randomizations (at a P-value $<=0.01$ ). Heat map representation of metabolic pathways was generated using the Mayday visualization tool version 2.12.

\section{Heterologous expression and purification of response regulator proteins}

Coding sequences corresponding to the $S$. mutans response regulators MbrC (SMU_1008) and VicR (SMU_ 1517c) were PCR-amplified with Phusion-Polymerase (NEB) using primer pairs MbrCFor/MbrCRev and VicRFor/VicRRev respectively (see Additional file 9). Homologous flanks to the vector sequence of pET28c were implemented at the 5 ' ends of the primer. The purified DNA was subsequently cloned in expression vector pET28c (Novagen) cut with NcoI/XhoI (Fermentas) using 
the CloneEZKit (Genescript). Resulting plasmids bearing the coding sequence for a $\mathrm{N}$-terminal His-Tag were verified by sequencing and transformed in BL21Star. Cells were grown in $4 \mathrm{l}$ shaking flaks, induced with $1 \mathrm{mM}$ IPTG at an $\mathrm{OD}_{600}$ of 0.5 and harvested 4 hours after induction. The resulting cell pellet was re-suspended in lysis buffer $(50 \mathrm{mM}$ Tris, $150 \mathrm{mM} \mathrm{NaCl}, \mathrm{pH}$ 7.4) supplemented with $4 \mathrm{mg} / \mathrm{ml}$ lysozyme (Sigma) and frozen at $-20^{\circ} \mathrm{C}$. After de-freezing the pellet, $100 \mu \mathrm{g}$ DNAse I (Roche) and $5 \mathrm{mM} \mathrm{MgSO}_{4}$ (Sigma, final concentration) were added and 5 cycles of sonification for 60 seconds were applied to lyse cells (duty cycle $0.5 \mathrm{Sec}$, pause $1 \mathrm{sec}$, amplitude 60\%). Finally $0.1 \%$ NP40 (Sigma, final concentration) and $10 \mathrm{mM}$ imidazol (Sigma, final concentration) were added and cell debris was removed by centrifugation and filtration $(0.45 \mu \mathrm{m})$. His-tagged proteins were purified on a HIS-Talon (Clontech) column using a Duo Flow FPLC (Biorad) at a flow rate of $0.5 \mathrm{ml} / \mathrm{h}$. Resulting purified proteins were desalted using PD10 columns (Amersham), eluted in 1xPBS buffer and measured using a spectrophotometer (Nanodrop ND1000, Peqlab) to determine the protein concentration. The response regulator was over 99\% pure as confirmed by SDS-PAGE (data not shown). Promoter regions containing the potential binding sites of $\mathrm{MbrC}$ were PCR amplified using Taq-polymerase. The primers used are listed in Additional file 9. PCR-products were purified using the PCR-Purification Kit (Qiagen) and used as targets for EMSA.

\section{Deletion of potential binding sites}

Potential binding sites of the response regulator $\mathrm{MbrC}$ were deleted using a PCR-driven overlap extension method [120]. Motifs in the promoter regions of SMU_610, SMU_718, and SMU_1006 were deleted by two initial PCRs generating overlapping DNA sequences. Taq polymerase (Qiagen) was used for the amplification of regions flanking the potential binding sites. PCR-products were purified from an agarose gel using the Gel Extraction Kit (Qiagen) and used as template for a third PCR with Phusion polymerase using primers (Additional file 9) spanning the whole region. Purified PCR-products were cloned blunt-end in the EcoRV restriction site of vector pGEM5Zf $(+)$ (Promega). Resulting plasmids were verified by sequencing and used as template for a PCR to finally generate the DNA-targets for EMSA.

\section{EMSA procedure}

The response regulator ( $\mathrm{MbrC}$ and $\mathrm{VicR})$ proteins were activated by acetylphosphate (Sigma). An aliqot of the protein was incubated for $2 \mathrm{~h}$ at RT (room temperature) in reaction buffer ( $25 \mathrm{mM}$ acetylphosphate, $50 \mathrm{mM}$ Tris- $\mathrm{HCl}, 50 \mathrm{mM} \mathrm{KCl}, 10 \mathrm{mM} \mathrm{MgCl}_{2}, 4 \mathrm{mM}$ dithiothreitol) as described previously. Excess of acetylphosphate was removed by filtration and the protein was serially diluted in binding buffer $(10 \mathrm{mM}$ Tris, $1 \mathrm{mM}$ EDTA, $100 \mathrm{mM} \mathrm{KCl}, 100 \mu \mathrm{M}$ DTT, $5 \% \mathrm{vol} / \mathrm{vol}$ glycerol, $10 \mu \mathrm{g} / \mathrm{ml} \mathrm{BSA}, \mathrm{pH} 7.5) .0 .5 \mathrm{pmol}$ of target and competitor DNA was added to each reaction and incubated for $1 \mathrm{~h}$ at RT. The unrelated response regulator VicR which has a similar MW and $\mathrm{pI}$ as the response regulator $\mathrm{MbrC}$ under study was used as a negative control to rule out unspecific DNA binding. Four $\mu \mathrm{l}$ of the reaction mixture was applied on a $5 \%$ acrylamide gel run in Tris-borate-EDTA (TBE) buffer at $\mathrm{pH}$ 7.4. Gels were stained using SybrGold and visualized in a transilluminator (Alpha DigiDoc, Biorad) at $254 \mathrm{~nm}$.

\section{Construction of gene deletion mutants and a cysR complementation strain}

Upstream and downstream flanking regions of $c y s R$ were PCR-amplified using primers CysR P1/2 and CysR P3/4 (see Additional file 9 for the primers used to generate deletion mutants) and genomic DNA of $S$. mutans UA159 as template. The erythromycin resistance cassette was amplified from genomic DNA of a previously constructed mutant [5]. Restriction sites of AscI and FseI were introduced via the 5'-termini of the primers. After restriction digestion of purified PCR-products with the appropriate restriction enzymes, the up and downstream flanks were ligated to the ERM cassette using T4 DNA Ligase and directly transformed in the S. mutans UA159 WT strain according to the procedure of Li et al. [121]. Gene deletion strains were selected on THBY agar plates containing $10 \mu \mathrm{g} / \mathrm{ml}$ erythromycin. Genomic DNA of isolated clones was analysed with PCR and primers P1 and $\mathrm{P} 4$ spanning the entire gene deletion construct. Sequencing was used to further confirm the correct insertion of the gene deletion construct via double homologous recombination. For the generation of the four other gene deletion strains $(g \ln R, f a b T, r g g$ and $s p x A)$, a similar procedure was carried out.

RNA was isolated from the exponentially growing cys $R$ gene deletion strain and was verified for the absence of cysR mRNA with RT-PCR using the 3 primer pairs Q-CYSR1-F/R, Q-CYSR2-F/R, and Q-CYSR3-F/R. Primers specific for the ComX (ComX F/R) and ComE (ComE F/R) encoding genes were used as positive controls for the RT-PCR reaction. Reactions in which the reverse transcriptase was replaced with water functioned as negative controls. For the complementation of the $c y s R$ gene deletion strain, the entire cysR gene including its promoter region were PCR-amplified with primers C-CYSR1-F and C-CYSR1-R and cloned blunt end via the SmaI restriction site in the replicative plasmid pDL278 [122]. Resulting plasmids were verified by sequencing and transformed in the cys $R$ gene deletion strain as described elsewhere [121]. 


\section{Viability measurements of the carolacton treated cys $R$ deletion strain using Live/Dead viability staining and by Cfu determination}

Viability measurements using live/dead viability staining of carolacton treated and untreated biofilms of the $S$. mutans UA159 wildtype, gene deletion and complementation strains were essentially performed as previously described [5]. Biofilms of S. mutans UA159 WT were used as positive control. The experiment was repeated in two biological replicates. For the cfu determination, biofilms representing one condition were harvested and pooled from 5 individual wells. Chains and biofilm clumps were dispersed and destroyed using mild ultrasonification conditions as described before [123] and plated on THBY-agar plates (containing $10 \mu \mathrm{g} / \mathrm{ml}$ erythromycin for the mutant) in 3 technical replicates. Live dead staining before and after sonification verified that the sonification had no significant influence on cell viability. The overall experiment was repeated in 2 biological replicates.

\section{Additional files}

Additional file 1: Functional enrichment analysis (with respect to biological functional classes and KEGG metabolic pathways) of the set of up- and downregulated GTAAs upon carolacton treatment at each time point. The biological functional classes derived from the Oralgen database (version 2011 and now re-named as The Bioinformatic Resource for Oral Pathogens) were classified as main or sub-functional classes depending on their annotation hierarchies whereas the pathway information was derived from the KEGG database (version 2011). Functional classes that are most significantly enriched (significance score $>=1$ ) are marked in dark green and those with decreasing significance with diminishing shades of green under the threshold limitation of significance score $>=0$. The hypergeometric distribution with Benjamini-Hochberg correction was used to determine the significance of over-representation. NA indicates the absence of GTAAs at the given $\log 2$-fold ratio cutoff or the absence of statistically significant over-representation (significance score $<0$ ) of functional categories.

Additional file 2: The $S$. mutans genetic co-expression network in response to carolacton treatment as inferred using the Trend Correlation method. 8284 gene pairs were inferred as statistically significant by using the standard performance metrics and procedures as described in the Methods section. The P-value indicates the randomness of the inferred gene-gene co-expression relationships. Positive co-expression relationships between genes are denoted by 1 and negative inverted relationships by 0 under the 'Relationship' column.

Additional file 3: The list of $S$. mutans UA159 transcription factors and their binding motif information compiled in this study. TF: Transcription factor. BS: Bacillus subtilis. AAA (SMART database accession number SM00382): Domain found in ATPases associated with a variety of cellular processes; Bac_DnaA_C (SMU00760): Domain representing the C-terminal regions of bacterial DnaA proteins; PDZ (SM00228): Domain representing distinct regions found in diverse signalling proteins; REC (SM00448): Type of response regulator domain; HTH_LUXR (SM00421): DNA-bending helix-turn-helix domain present in transcriptional regulators of the LuxR/FixJ family of response regulators. HTH_MERR (SM00422): Domain found in transcriptional regulators which bind DNA via a helix-turn-helix structure and which mediate mercurydependent induction of the mercury-resistance operon. CoA_binding (SM00881): Domain found in a number of proteins including succinyl CoA synthases, malate and ATP-citrate ligases. HTH_ARSR (SM00418): DNA-binding, winged helix-turn-helix (WHTH) domain present in transcription regulators of the arsR/smtB family and involved in stress- response to heavy metal ions; Trans_Reg_C (SM00862): Domain found in the C-terminal regions of response regulator proteins.

Additional file 4: The list of regulator-target gene relationships based on the prediction of binding sites using Pattern Matching. Please refer to the Methods section for more information on the Pattern Matching procedure.

Additional file 5: Data representing the transcriptional regulatory response network (TRRN) of $S$. mutans biofilms upon treatment with the biofilm inhibitor carolacton. The table displays the co-expression characteristics of significant correlations as well as the information corresponding to the predicted transcription factor binding sites on the upstream regulatory regions of the target genes or their corresponding operons. NA*: motifs predicted using the pattern matching tool dna pattern. Positive relationship between the genes is denoted by 1 and negative inverted relationships by 0 under the Relationship column.

Additional file 6: The different types of local network motifs identified in the $S$. mutans TRRN upon carolacton treatment. The motifs were identified and their corresponding statistical scores determined using the MFINDER tool $[47,119]$ after 10000 randomizations. *SD denotes the standard deviation of the number of occurrences of the motif in randomized networks. $\wedge$ The "Z score" is a measure of the statistical significance of the motifs and is determined as $\left(M_{\text {real }}-M_{\text {rand }}\right) /$ SD. Motifs with P-values $<=0.01$ were considered as significant.

Additional file 7: List of enriched categories in the co-regulated gene groups or subnetworks of the S. mutans TRRN upon carolacton treatment. Over-represented categories include KEGG metabolic pathways (KEGG MP), Biological functional classes (BFC) and gene ontology terms (GO). The details pertaining to the calculation of the significance scores are provided under the Methods section.

Additional file 8: The normalized expression profiles of the genes co-regulated by the global transcription factors CcpA and CodY. For the CodY co-regulated gene group, only the expression profiles of genes with positive relationships are shown.

Additional file 9: Primers used in the study.

\section{Abbreviations}

TRRN: Transcriptional regulatory response network; EMSA: Electro mobility shift assay; TCS: Two component system; RR: Response regulator; gtf: glucosyltransferase; gbpB: glucan binding protein B; TSNI: Time series network identification; GTAA: Genes corresponding to transcripts with altered abundances; KEGG: Kyoto encyclopedia of genes and genomes; TC Method: Trend Correlation method; ORF: Open reading frame; PATRIC: Pathosystems resource integratrion center; WT: Wild type; RT-PCR: Reverse transcriptase polymerase chain reaction; SDS-PAGE: Sodium Do-decyl Sulfate Poly-Acrylamide Gel Electrophoresis; PSSM: Position specific scoring matrix; RSAT: Regulatory sequence analysis tools; GTP: Guanosine tri phosphate; SIM: Single input module; MIM: Multiple input module; FFL: Feed forward loop; MOFFL: Multi-output Ffl; UDP: Uridine di phosphate; UTP: Uridine tri phosphate; UMP: Uridine mono phosphate; UDP-N-AG: Uridine Di-Phosphate N-Acetyl Glucosamine; ATR: Acid tolerance response; CFU: Colony forming units; LTTR: LysR type transcriptional regulators; XIP: sigX inducing peptide; OAS: O-acetylserine.

\section{Competing interests}

The authors declare that they have no competing interests.

\section{Authors' contributions}

PS carried out the major reverse engineering and network analysis and contributed significantly to drafting the manuscript. MR carried out the experimental work for generating the microarray data and EMSA studies and performed the primary and secondary analysis of the microarray data. WW participated in the conception and coordination of the study and contributed to drafting the manuscript. FH contributed to developing the reverse engineering method and its application and revised the manuscript. IWD contributed to the microbial aspects and valuable discussions. AZE conceived of and supervised the study and revised the manuscript. All authors read and approved the final manuscript. 


\section{Acknowledgement}

This study was done within the project "Development of biofilm inhibitors using a systems biology approach" (0315411) which is financed by the German Federal Ministry of Education and Research (BMBF) in the frame of the Research Program "Medical systems biology - MedSys".

\section{Data availability}

The time-series microarray data set generated as part of this study is available in the Gene Expression Omnibus (GEO) repository [accession id GSE53264].

\section{Author details}

${ }^{1}$ Institute of Bioprocess and Biosystems Engineering, Hamburg University of Technology, 21073 Hamburg, Germany. ${ }^{2}$ Research Group Microbial Communication, Helmholtz Center for Infection Research, Inhoffenstrasse 7, 38124 Braunschweig, Germany. ${ }^{3}$ Luxembourg Centre for Systems Biomedicine, 7, Avenue des Hauts Fourneaux, L-4362 Belval, Luxembourg.

Received: 27 November 2013 Accepted: 17 April 2014

Published: 12 May 2014

\section{References}

1. Stoodley P, Sauer K, Davies DG, Costerton JW: Biofilms as complex differentiated communities. Annu Rev Microbiol 2002, 56:187-209.

2. Dufour $D$, Leung $V$, Levesque $C M$ : Bacterial biofilm: structure, function, and antimicrobial resistance. Endo Topics 2012, 22(1):2-16.

3. Kunze B, Reck M, Dötsch A, Lemme A, Schummer D, Irschik H, Steinmetz H, Wagner-Döbler I: Damage of Streptococcus mutans biofilms by carolacton, a secondary metabolite from the myxobacterium Sorangium cellulosum. BMC Microbio/ 2010, 10:199.

4. Roychoudhury S, Zielinski NA, Ninfa AJ, Allen NE, Jungheim LN, Nicas TI, Chakrabarty AM: Inhibitors of two-component signal transduction systems: inhibition of alginate gene activation in Pseudomonas aeruginosa. Proc Nat/ Acad Sci U S A 1993, 90:965-969.

5. Reck M, Rutz K, Kunze B, Tomasch J, Surapaneni SK, Schulz S, Wagner-Döbler I: The biofilm inhibitor carolacton disturbs membrane integrity and cell division of Streptococcus mutans through the serine/threonine protein kinase PknB. J Bacterio/ 2011, 193:5692-5706.

6. Li Y, Tang N, Aspiras MB, Lau PCY, Lee JH, Ellen RP, Cvitkovitch DG: A quorum-sensing signaling system essential for genetic competence in Streptococcus mutans is involved in biofilm formation. J Bacterio/ 2002, 184:2699-2708.

7. Qi F, Kreth J, Lévesque CM, Kay O, Mair RW, Shi W, Cvitkovitch DG, Goodman SD: Peptide pheromone induced cell death of Streptococcus mutans. FEMS Microbiol Lett 2005, 251:321-326.

8. Bhagwat SP, Nary J, Burne RA: Effects of mutating putative twocomponent systems on biofilm formation by Streptococcus mutans UA159. FEMS Microbiol Lett 2001, 205:225-230.

9. Sasková L, Nováková L, Basler M, Branny P: Eukaryotic-type serine/ threonine protein kinase StkP is a global regulator of gene expression in Streptococcus pneumoniae. J Bacterio/ 2007, 189:4168-4179.

10. He F, Balling $R$, Zeng $A$ : Reverse engineering and verification of gene networks:principles, assumptions, and limitations of present methods and future perspectives. J Biotechnol 2009, 144:190-203.

11. Cabusora L, Sutton E, Fulmer A, Forst CV: Differential network expression during drug and stress response. Bioinformatics 2005, 21:2898-2905.

12. Bansal M, Della Gatta G, Di Bernardo D: Inference of gene regulatory networks and compound mode of action from time course gene expression profiles. Bioinformatics 2006, 22:815-822.

13. Aluru M, Zola J, Nettleton D, Aluru S: Reverse engineering and analysis of large genome-scale gene networks. Nucleic Acids Res 2013, 41:e24.

14. Faith JJ, Hayete B, Thaden JT, Mogno I, Wierzbowski J, Cottarel G, Kasif S, Collins JJ, Gardner TS: Large-scale mapping and validation of Escherichia coli transcriptional regulation from a compendium of expression profiles. PLOS Biol 2007, 5:e8.

15. He F, Chen H, Probst-Kepper M, Geffers R, Eifes S, Del Sol A, Schughart K, Zeng $A$, Balling R: PLAU inferred from a correlation network is critical for suppressor function of regulatory T cells. Mol Syst Biol 2012, 8:624.

16. Faria JP, Overbeek R, Xia F, Rocha M, Rocha I, Henry CS: Genome-scale bacterial transcriptional regulatory networks: reconstruction and integrated analysis with metabolic models. Brief Bioinform 2013. Epub ahead of print. doi: 10.1093/bib/bbs071.

17. Fadda A, Fierro AC, Lemmens K, Monsieurs P, Engelen K, Marchal K: Inferring the transcriptional network of Bacillus subtilis. Mol Biosyst 2009, 5:1840-1852.

18. Castro-Melchor M, Charaniya S, Karypis G, Takano E, Hu W: Genome-wide inference of regulatory networks in Streptomyces coelicolor. BMC Genomics 2010, 11:578.

19. Bonneau R, Reiss DJ, Shannon P, Facciotti M, Hood L, Baliga NS, Thorsson V: The Inferelator: an algorithm for learning parsimonious regulatory networks from systems-biology data sets de novo. Genome Biol 2006, 7:R36.

20. Fu Y, Jarboe LR, Dickerson JA: Reconstructing genome-wide regulatory network of E. coli using transcriptome data and predicted transcription factor activities. BMC Bioinformatics 2011, 12:233.

21. Guelzim N, Bottani S, Bourgine P, Képès F: Topological and causal structure of the yeast transcriptional regulatory network. Nat Genet 2002, 31:60-63.

22. Altwasser R, Linde J, Buyko E, Hahn U, Guthke R: Genome-Wide Scale-Free Network Inference for Candida albicans. Front Microbiol 2012, 3:51.

23. He FQ, Wang W, Zheng P, Sudhakar P, Sun J, Zeng AP: Essential O2-responsive genes of Pseudomonas aeruginosa and their network revealed by integrating dynamic data from inverted conditions. Integr Biol 2014, 6:215-223.

24. Bailly-Bechet M, Braunstein A, Pagnani A, Weigt M, Zecchina R: Inference of sparse combinatorial-control networks from gene-expression data: a message passing approach. BMC Bioinformatics 2010, 11:355.

25. Basso K, Margolin A, Stolovitzky G, Klein U, Dalla-Favera R, Califano A: Reverse engineering of regulatory networks in human B cells. Nat Genet 2005, 37:382-390.

26. O'Rourke KP, Shaw JD, Pesesky MW, Cook BT, Roberts SM, Bond JP, Spatafora GA: Genome-wide characterization of the SloR metalloregulome in Streptococcus mutans. J Bacteriol 2010, 192:1433-1443.

27. Ahn S, Wen ZT, Burne RA: Effects of oxygen on virulence traits of Streptococcus mutans. J Bacterio/ 2007, 189:8519-8527.

28. Ajdić D, Pham VIT: Global transcriptional analysis of Streptococcus mutans sugar transporters using microarrays. J Bacterio/ 2007, 189:5049-5059.

29. Liu J, Wu C, Huang I, Merritt J, Qi F: Differential response of Streptococcus mutans towards friend and foe in mixed-species cultures. Microbiology 2011, 157:2433-2444.

30. Lévesque CM, Mair RW, Perry JA, Lau PCY, Li Y, Cvitkovitch DG: Systemic inactivation and phenotypic characterization of two-component systems in expression of Streptococcus mutans virulence properties. Lett Appl Microbiol 2007, 45:398-404

31. Perry JA, Lévesque CM, Suntharaligam P, Mair RW, Bu M, Cline RT, Peterson SN, Cvitkovitch DG: Involvement of Streptococcus mutans regulator RR11 in oxidative stress response during biofilm growth and in the development of genetic competence. Lett Appl Microbiol 2008, 47:439-444.

32. He F, Zeng $A$ : In search of functional association from time-series microarray data based on the change trend and level of gene expression. BMC Bioinformatics 2006, 7:69.

33. Rodionov DA: Comparative genomic reconstruction of transcriptional regulatory networks in bacteria. Chem Rev 2007, 107:3467-3497.

34. Leyn SA, Kazanov MD, Sernova NV, Ermakova EO, Novichkov PS, Rodionov DA: Genomic Reconstruction of Transcriptional Regulatory Network in Bacillus subtilis. J Bacterio/ 2013, 195:2463-2473.

35. Cornish JP, Matthews F, Thomas JR, Erill I: Inference of self-regulated transcriptional networks by comparative genomics. Evol Bioinform Online 2012, 8:449-461

36. Novichkov PS, Laikova ON, Novichkova ES, Gelfand MS, Arkin AP, Dubchak I, Rodionov DA: RegPrecise: a database of curated genomic inferences of transcriptional regulatory interactions in prokaryotes. Nucleic Acids Res 2010, 38:D111-8.

37. Medina-Rivera A, Abreu-Goodger C, Thomas-Chollier M, Salgado H, Collado-Vides van Helden J: Theoretical and empirical quality assessment of transcription factor-binding motifs. Nucleic Acids Res 2011, 39:808-824.

38. Lemos JA, Nascimento MM, Lin VK, Abranches J, Burne RA: Global regulation by (p)ppGpp and CodY in Streptococcus mutans. J Bacteriol 2008, 190:5291-5299. 
39. Isalan M, Lemerle C, Michalodimitrakis K, Horn C, Beltrao P, Raineri E, Garriga-Canut M, Serrano L: Evolvability and hierarchy in rewired bacterial gene networks. Nature 2008, 452:840-845

40. $\mathrm{Ma} \mathrm{H}$, Buer J, Zeng $\mathrm{A}$ : Hierarchical structure and modules in the Escherichia coli transcriptional regulatory network revealed by a new top-down approach. BMC Bioinformatics 2004, 5:199.

41. Galán-Vásquez E, Luna B, Martínez-Antonio A: The Regulatory Network of Pseudomonas aeruginosa. Microb Inform Exp 2011, 1:3.

42. Dufour YS, Kiley PJ, Donohue TJ: Reconstruction of the core and extended regulons of global transcription factors. PLoS Genet 2010, 6:e1001027.

43. Chaturongakul S, Raengpradub S, Palmer ME, Bergholz TM, Orsi RH, Hu Y, Ollinger J, Wiedmann M, Boor KJ: Transcriptomic and phenotypic analyses identify coregulated, overlapping regulons among PrfA, CtsR, HrcA, and the alternative sigma factors sigmaB, sigmaC, sigmaH, and sigmaL in Listeria monocytogenes. App/ Environ Microbiol 2011, 77:187-200.

44. Konagurthu AS, Lesk AM: Single and multiple input modules in regulatory networks. Proteins 2008, 73:320-324.

45. Mangan S, Alon U: Structure and function of the feed-forward loop network motif. Proc Natl Acad. Sci. U.S.A. 2003, 100:11980-11985.

46. Kalir S, McClure J, Pabbaraju K, Southward C, Ronen M, Leibler S, Surette $\mathrm{MG}$, Alon U: Ordering genes in a flagella pathway by analysis of expression kinetics from living bacteria. Science 2001, 292:2080-2083.

47. Kashtan N, Itzkovitz S, Milo R, Alon U: Topological generalizations of network motifs. Phys Rev E Stat Nonlin Soft Matter Phys 2004, 70:31909.

48. Ma H, Kumar B, Ditges U, Gunzer F, Buer J, Zeng A: An extended transcriptional regulatory network of Escherichia coli and analysis of its hierarchical structure and network motifs. Nucleic Acids Res 2004, 32:6643-6649.

49. Chen P, Chen YM, Yu S, Sher S, Lai C, Chia J: Role of GInR in acid-mediated repression of genes encoding proteins involved in glutamine and glutamate metabolism in Streptococcus mutans. Appl Environ Microbiol 2010, 76:2478-2486.

50. Ouyang J, Tian X, Versey J, Wishart A, Li Y: The BceABRS four-component system regulates the bacitracin-induced cell envelope stress response in Streptococcus mutans. Antimicrob Agents Chemother 2010, 54:3895-3906.

51. Abranches J, Nascimento MM, Zeng L, Browngardt CM, Wen ZT, Rivera MF, Burne RA: CcpA regulates central metabolism and virulence gene expression in Streptococcus mutans. J Bacteriol 2008, 190:2340-2349.

52. Abranches J, Chen YM, Burne RA: Characterization of Streptococcus mutans strains deficient in EIIAB Man of the sugar phosphotransferase system. Appl Environ Microbiol 2003, 69:4760-4769.

53. Zeng $L$, Wen $Z T$, Burne RA: A novel signal transduction system and feedback loop regulate fructan hydrolase gene expression in Streptococcus mutans. Mol Microbiol 2006, 62:187-200.

54. Zeng L, Das S, Burne RA: Genetic analysis of the functions and interactions of components of the LevQRST signal transduction complex of Streptococcus mutans. PLoS One 2011, 6:e17335.

55. Webb AJ, Homer KA, Hosie AHF: Two closely related ABC transporters in Streptococcus mutans are involved in disaccharide and/or oligosaccharide uptake. J Bacterio/ 2008, 190:168-178.

56. Loesche WJ: Role of Streptococcus mutans in human dental decay. Microbiol Rev 1986, 50:353-380.

57. Touger-Decker R, van Loveren C: Sugars and dental caries. Am J Clin Nutr 2003, 78:881S-892S.

58. van Houte J, Upeslacis VN, Jordan HV, Skobe Z, Green DB: Role of sucrose in colonization of Streptococcus mutans in conventional SpragueDawley rats. J Dent Res 1976, 55:202-215.

59. Cury JA, Francisco SB, Del Bel Cury AA, Tabchoury CP: In situ study of sucrose exposure, mutans streptococci in dental plaque and dental caries. Braz Dent J 2001, 12:101-104.

60. Nobre dos Santos M, Melo dos Santos L, Francisco SB, Cury JA: Relationship among dental plaque composition, daily sugar exposure and caries in the primary dentition. Caries Res 2002, 36:347-352.

61. Sadykov MR, Mattes TA, Luong TT, Zhu Y, Day SR, Sifri CD, Lee CY, Somerville GA: Tricarboxylic acid cycle-dependent synthesis of Staphylococcus aureus Type 5 and 8 capsular polysaccharides. J Bacteriol 2010, 192:1459-1462.

62. Howden BP, McEvoy CRE, Allen DL, Chua K, Gao W, Harrison PF, Bell J, Coombs G, Bennett-Wood V, Porter JL, Robins-Browne R, Davies JK, Seemann T, Stinear TP: Evolution of multidrug resistance during Staphylococcus aureus infection involves mutation of the essential two component regulator WaIKR. PLoS Pathog 2011, 7:e1002359.
63. Dashper SG, Riley PF, Reynolds EC: Characterization of glutamine transport in Streptococcus mutans. Oral Microbiol Immunol 1995, 10:183-187.

64. Cvitkovitch DG, Gutierrez JA, Bleiweis AS: Role of the citrate pathway in glutamate biosynthesis by Streptococcus mutans. J Bacterio/ 1997, 179:650-655.

65. Yu H, Yao Y, Liu Y, Jiao R, Jiang W, Zhao G: A complex role of Amycolatopsis mediterranei GlnR in nitrogen metabolism and related antibiotics production. Arch Microbiol 2007, 188:89-96.

66. Rolerson E, Swick A, Newlon L, Palmer C, Pan Y, Keeshan B, Spatafora G: The SloR/Dlg metalloregulator modulates Streptococcus mutans virulence gene expression. J Bacteriol 2010, 192:1433-1443.

67. Chattoraj P, Mohapatra SS, Rao JLUM, Biswas I: Regulation of transcription by SMU.1349, a TetR family regulator, in Streptococcus mutans. $J$ Bacteriol 2011, 193:6605-6613.

68. Kreth J, Hung DCl, Merritt J, Perry J, Zhu L, Goodman SD, Cvitkovitch DG, Shi W, Qi F: The response regulator ComE in Streptococcus mutans functions both as a transcription activator of mutacin production and repressor of CSP biosynthesis. Microbiology 2007, 153:1799-1807.

69. Krastel K, Senadheera DB, Mair R, Downey JS, Goodman SD, Cvitkovitch DG: Characterization of a glutamate transporter operon, glnQHMP, in Streptococcus mutans and its role in acid tolerance. J Bacteriol 2010, 192:984-993.

70. Matsui R, Cvitkovitch D: Acid tolerance mechanisms utilized by Streptococcus mutans. Future Microbiol 2010, 5:403-417.

71. Svensäter G, Larsson UB, Greif EC, Cvitkovitch DG, Hamilton IR: Acid tolerance response and survival by oral bacteria. Oral Microbiol Immunol 1997, 12:266-273.

72. Liu Y, Burne RA: Multiple two-component systems of Streptococcus mutans regulate agmatine deiminase gene expression and stress tolerance. J Bacteriol 2009, 191:7363-7366.

73. Lee SF, Delaney GD, Elkhateeb M: A two-component covRS regulatory system regulates expression of fructosyltransferase and a novel extracellular carbohydrate in Streptococcus mutans. Infect Immun 2004, 72:3968-3973.

74. Deng DM, Liu MJ, Cate JM t, Crielaard W: The VicRK system of Streptococcus mutans responds to oxidative stress. J Dent Res 2007, 86:606-610.

75. Tremblay YDN, Lo H, Li Y, Halperin SA, Lee SF: Expression of the Streptococcus mutans essential two-component regulatory system VicRK is $\mathrm{pH}$ and growth-phase dependent and controlled by the LiaFSR threecomponent regulatory system. Microbiology 2009, 155:2856-2865.

76. Senadheera MD, Guggenheim B, Spatafora GA, Huang YC, Choi J, Hung DCI, Treglown JS, Goodman SD, Ellen RP, Cvitkovitch DG: A VicRK signal transduction system in Streptococcus mutans affects gtfBCD, gbpB, and $\mathrm{ftf}$ expression, biofilm formation, and genetic competence development. J Bacteriol 2005, 187:4064-4076.

77. Senadheera D, Krastel K, Mair R, Persadmehr A, Abranches J, Burne RA, Cvitkovitch DG: Inactivation of VicK affects acid production and acid survival of Streptococcus mutans. J Bacteriol 2009, 191:6415-6424.

78. Lévesque CM, Voronejskaia E, Huang YC, Mair RW, Ellen RP, Cvitkovitch DG: Involvement of sortase anchoring of cell wall proteins in biofilm formation by Streptococcus mutans. Infect Immun 2005, 73:3773-3777.

79. Duque C, Stipp RN, Wang B, Smith DJ, Höfling JF, Kuramitsu HK, Duncan MJ, Mattos-Graner RO: Downregulation of GbpB, a component of the VicRK regulon, affects biofilm formation and cell surface characteristics of Streptococcus mutans. Infect Immun 2011, 79:786-796.

80. Senadheera DB, Cordova M, Ayala EA, LE de Chávez P, Singh K, Downey JS, Svensäter G, Goodman SD, Cvitkovitch DG: Regulation of bacteriocin production and cell death by the VicRK signaling system in Streptococcus mutans. J Bacterio/ 2012, 194:1307-1316.

81. Sperandio B, Gautier C, Pons N, Ehrlich DS, Renault P, Guédon E: Three paralogous LysR-type transcriptional regulators control sulfur amino acid supply in Streptococcus mutans. J Bacteriol 2010, 192:3464-3473.

82. Fleuchot B, Gitton C, Guillot A, Vidic J, Nicolas P, Besset C, Fontaine L, Hols P, Leblond-Bourget N, Monnet V, Gardan R: Rgg proteins associated with internalized small hydrophobic peptides: a new quorum-sensing mechanism in streptococci. Mol Microbiol 2011, 80:1102-1119.

83. Filipe SR, Severina E, Tomasz A: Functional analysis of Streptococcus pneumoniae MurM reveals the region responsible for its specificity in the synthesis of branched cell wall peptides. J Biol Chem 2001, 276:39618-39628. 
84. Filipe SR, Severina E, Tomasz A: The role of murMN operon in penicillin resistance and antibiotic tolerance of Streptococcus pneumoniae. Microb Drug Resist 2001, 7:303-316.

85. Filipe SR, Severina E, Tomasz A: The murMN operon: a functional link between antibiotic resistance and antibiotic tolerance in Streptococcus pneumoniae. Proc Natl Acad Sci U S A 2002, 99:1550-1555.

86. Filipe SR, Tomasz A: Inhibition of the expression of penicillin resistance in Streptococcus pneumoniae by inactivation of cell wall muropeptide branching genes. Proc Natl Acad Sci U S A 2000, 97:4891-4896.

87. Typas A, Banzhaf M, Gross CA, Vollmer W: From the regulation of peptidoglycan synthesis to bacterial growth and morphology. Nat Rev Microbiol 2012, 10:123-136.

88. de Jonge BLM, Gage D, Xu N: The carboxyl terminus of peptidoglycan stem peptides is a determinant for methicillin resistance in Staphylococcus aureus. Antimicrob Agents Chemother 2002, 46:3151-3155.

89. Garcia-Bustos JF, Chait BT, Tomasz A: Altered peptidoglycan structure in a pneumococcal transformant resistant to penicillin. J Bacteriol 1988, 170:2143-2147.

90. Garcia-Bustos J, Tomasz A: A biological price of antibiotic resistance: major changes in the peptidoglycan structure of penicillin-resistant pneumococci. Proc Natl Acad Sci U S A 1990, 87:5415-5419.

91. Simelyte E, Rimpiläinen M, Zhang X, Toivanen P: Role of peptidoglycan subtypes in the pathogenesis of bacterial cell wall arthritis. Ann Rheum Dis 2003, 62:976-982.

92. Kajfasz JK, Rivera-Ramos I, Abranches J, Martinez AR, Rosalen PL, Derr AM, Quivey RG, Lemos JA: Two Spx proteins modulate stress tolerance, survival, and virulence in Streptococcus mutans. J Bacteriol 2010, 192:2546-2556.

93. Sperandio B, Gautier C, McGovern S, Ehrlich DS, Renault P, Martin-Verstraete I, Guédon E: Control of methionine synthesis and uptake by MetR and homocysteine in Streptococcus mutans. J Bacteriol 2007, 189:7032-7044.

94. Catt DM, Gregory RL: Streptococcus mutans murein hydrolase. J Bacteriol 2005, 187:7863-7865.

95. Dufour D, Lévesque CM: Cell death of Streptococcus mutans induced by a quorum-sensing peptide occurs via a conserved streptococcal autolysin. J Bacterio/ 2013, 195:105-114.

96. Huynen M, Snel B, Lathe W, Bork P: Predicting protein function by genomic context: quantitative evaluation and qualitative inferences. Genome Res 2000, 10:1204-1210.

97. Wu H, Su Z, Mao F, Olman V, Xu Y: Prediction of functional modules based on comparative genome analysis and Gene Ontology application. Nucleic Acids Res 2005, 33:2822-2837.

98. Mavromatis K, Chu K, Ivanova N, Hooper SD, Markowitz VM, Kyrpides NC: Gene context analysis in the Integrated Microbial Genomes (IMG) data management system. PLoS One 2009, 4:e7979.

99. Allen JP, Neely MN: CpsY influences Streptococcus iniae cell wall adaptations important for neutrophil intracellular survival. Infect Immun 2012, 80:1707-1715.

100. Federle MJ, Morrison DA: One if by land, two if by sea: signalling to the ranks with CSP and XIP. Mol Microbiol 2012, 86:241-245.

101. Mashburn-Warren L, Morrison DA, Federle MJ: A novel double-tryptophan peptide pheromone controls competence in Streptococcus spp. via an Rgg regulator. Mol Microbiol 2010, 78:589-606.

102. Beilharz K, Nováková L, Fadda D, Branny P, Massidda O, Veening J: Control of cell division in Streptococcus pneumoniae by the conserved Ser/Thr protein kinase StkP. Proc Natl Acad Sci U S A 2012, 109:E905-13.

103. Banu LD, Conrads G, Rehrauer H, Hussain H, Allan E, van der Ploeg JR: The Streptococcus mutans serine/threonine kinase, $\mathrm{PknB}$, regulates competence development, bacteriocin production, and cell wall metabolism. Infect Immun 2010, 78:2209-2220.

104. Hussain H, Branny P, Allan E: A eukaryotic-type serine/threonine protein kinase is required for biofilm formation, genetic competence, and acid resistance in Streptococcus mutans. J Bacteriol 2006, 188:1628-1632.

105. Güell M, Yus E, Lluch-Senar M, Serrano L: Bacterial transcriptomics: what is beyond the RNA horiz-ome? Nat Rev Microbiol 2011, 9:658-669.

106. Bhatt S, Romeo T, Kalman D: Honing the message: post-transcriptional and post-translational control in attaching and effacing pathogens. Trends Microbiol 2011, 19:217-224.

107. Koyanagi S, Lévesque CM: Characterization of a Streptococcus mutans intergenic region containing a small toxic peptide and its cis-encoded antisense small RNA antitoxin. PLoS One 2013, 8:e54291.
108. Hasona A, Crowley PJ, Levesque CM, Mair RW, Cvitkovitch DG, Bleiweis AS, Brady LJ: Streptococcal viability and diminished stress tolerance in mutants lacking the signal recognition particle pathway or YidC2. Proc Natl Acad Sci U S A 2005, 102:17466-17471.

109. Hasona A, Zuobi-Hasona K, Crowley PJ, Abranches J, Ruelf MA, Bleiweis AS, Brady LJ: Membrane composition changes and physiological adaptation by Streptococcus mutans signal recognition particle pathway mutants. J Bacteriol 2007, 189:1219-1230

110. Karp PD, Paley SM, Krummenacker M, Latendresse M, Dale JM, Lee TJ, Kaipa P, Gilham F, Spaulding A, Popescu L, Altman T, Paulsen I, Keseler IM, Caspi R: Pathway Tools version 13.0: integrated software for pathway/genome informatics and systems biology. Brief Bioinform 2010, 11:40-79.

111. Ideker T, Krogan NJ: Differential network biology. Mol Syst Biol 2012, 8:565.

112. Valcárcel B, Würtz $P$, al Seich Basatena N, Tukiainen T, Kangas AJ, Soininen $P$, Järvelin M, Ala-Korpela M, Ebbels TM, de lorio M: A differential network approach to exploring differences between biological states: an application to prediabetes. PLoS One 2011, 6:e24702.

113. Sierro N, Makita Y, de Hoon M, Nakai K: DBTBS: a database of transcriptional regulation in Bacillus subtilis containing upstream intergenic conservation information. Nucleic Acids Res 2008, 36:D93-96.

114. Li L, Stoeckert CJ, Roos DS: OrthoMCL: identification of ortholog groups for eukaryotic genomes. Genome Res 2003, 13:2178-2189.

115. Thomas-Chollier M, Sand O, Turatsinze J, Janky R, Defrance M, Vervisch E, Brohée S, van Helden J: RSAT: regulatory sequence analysis tools. Nucleic Acids Res 2008, 36:W119-27.

116. Turatsinze J, Thomas-Chollier M, Defrance M, van Helden J: Using RSAT to scan genome sequences for transcription factor binding sites and cis-regulatory modules. Nat Protoc 2008, 3:1578-1588.

117. Gillespie JJ, Wattam AR, Cammer SA, Gabbard JL, Shukla MP, Dalay O, Driscoll T, Hix D, Mane SP, Mao C, Nordberg EK, Scott M, Schulman JR, Snyder EE, Sullivan DE, Wang C, Warren A, Williams KP, Xue T, Yoo HS, Zhang C, Zhang Y, Will R, Kenyon RW, Sobral BW: PATRIC: the comprehensive bacterial bioinformatics resource with a focus on human pathogenic species. Infect Immun 2011, 79:4286-4298.

118. Smoot ME, Ono K, Ruscheinski J, Wang P, Ideker T: Cytoscape 2.8: new features for data integration and network visualization. Bioinformatics 2011, 27:431-432.

119. Kashtan N, Itzkovitz S, Milo R, Alon U: Efficient sampling algorithm for estimating subgraph concentrations and detecting network motifs. Bioinformatics 2004, 20:1746-1758.

120. Heckmann $\mathrm{KL}$, Pease LR: Gene splicing and mutagenesis by PCR-driven overlap extension. Nat Protoc 2007, 2:924-932.

121. Li YH, Lau PC, Lee JH, Ellen RP, Cvitkovitch DG: Natural genetic transformation of Streptococcus mutans growing in biofilms. J Bacteriol 2001, 183:897-908.

122. Dunny GM, Lee LN, LeBlanc DJ: Improved electroporation and cloning vector system for gram-positive bacteria. Appl Environ Microbiol 1991, 57:1194-1201.

123. Lemme A, Gröbe L, Reck M, Tomasch J, Wagner-Döbler I: Subpopulationspecific transcriptome analysis of competence-stimulating-peptideinduced Streptococcus mutans. J Bacterio/ 2011, 193:1863-1877.

\section{doi:10.1186/1471-2164-15-362}

Cite this article as: Sudhakar et al:: Construction and verification of the transcriptional regulatory response network of Streptococcus mutans upon treatment with the biofilm inhibitor carolacton. BMC Genomics 2014 15:362. 